\title{
Superconducting pairing and density-wave instabilities in quasi-one-dimensional conductors
}

\author{
J. C. Nickel, ${ }^{1,2}$ R. Duprat, ${ }^{1}$ C. Bourbonnais, ${ }^{1}$ and N. Dupuis ${ }^{2,3}$ \\ ${ }^{1}$ Regroupement Québecois sur les Matériaux de Pointe, Département de physique, \\ Université de Sherbrooke, Sherbrooke, Québec, Canada, J1K-2R1 \\ ${ }^{2}$ Laboratoire de Physique des Solides, CNRS UMR 8502, Université Paris-Sud, 91405 Orsay, France \\ ${ }^{3}$ Department of Mathematics, Imperial College, 180 Queen's Gate, London SW7 2AZ, UK
}

(Dated: October 25, 2005)

\begin{abstract}
Using a renormalization group approach, we determine the phase diagram of an extended quasione-dimensional electron gas model that includes interchain hopping, nesting deviations and both intrachain and interchain repulsive interactions. $d$-wave superconductivity, which dominates over the spin-density-wave (SDW) phase at large nesting deviations, becomes unstable to the benefit of a triplet $f$-wave phase for a weak repulsive interchain backscattering term $g_{1}^{\perp}>0$, despite the persistence of dominant SDW correlations in the normal state. Antiferromagnetism becomes unstable against the formation of a charge-density-wave state when $g_{1}^{\perp}$ exceeds some critical value. While these features persist when both Umklapp processes and interchain forward scattering $\left(g_{2}^{\perp}\right)$ are taken into account, the effect of $g_{2}^{\perp}$ alone is found to frustrate nearest-neighbor interchain $d$ and $f$-wave pairing and instead favor next-nearest-neighbor interchain singlet or triplet pairing. We argue that the close proximity of SDW and charge-density-wave phases, singlet $d$-wave and triplet $f$-wave superconducting phases in the theoretical phase diagram provides a possible explanation for recent puzzling experimental findings in the Bechgaard salts, including the coexistence of SDW and charge-density-wave phases and the possibility of a triplet pairing in the superconducting phase.
\end{abstract}

PACS numbers: 71.10.Li,74.20.Mn,74.70.Kn

\section{INTRODUCTION}

The theory of low-dimensional metals has exerted a strong influence on our understanding of ordered phases in quasi-one-dimensional (quasi-1D) organic conductors. The description of the extensively studied Bechgaard salts series $\left((\mathrm{TMTSF})_{2} \mathrm{X}\right)$ and their sulfur analogs, the Fabre $\left((\mathrm{TMTTF})_{2} \mathrm{X}\right)$ salts, has served over more than two decades to illustrate this view $\frac{1.2 .3}{1}$ For these materials, a direct correspondence can be traced between the various modulated spin and charge ordered states of their phase diagram and the possible states of the quasi-1D electron gas model when the couplings are repulsive and the filling of the band is commensurate with the underlying lattice ${ }^{4.5} \mathrm{In}$ its standard form, this generic model is defined by the phenomenological expression of the direct interaction between electrons in terms of weak intrachain backward $\left(g_{1}\right)$ and forward $\left(g_{2}\right)$ electron-electron scattering processes, to which Umklapp $\left(g_{3}\right)$ scattering amplitudes are added at commensurate band filling. ${ }^{5,6.7 .8}$ The quasi-1D character of the model is defined by adding an interchain single electron hopping integral $t_{\perp}$, which is at least an order of magnitude smaller than its longitudinal counterpart.

In virtually all compounds of the above series, superconductivity is observed next to a spin-density-wave state for some critical value on the pressure scale, 9.10 .11 .12 whereas antiferromagnetic spin correlations are found to dominate the metallic state precursor to superconductivity over a wide range of temperatures ${ }^{13.14}$ On the theoretical side, however, for spin-independent repulsive couplings and for a Fermi surface with good nesting prop- erties, the coexistence of spin-density-wave (SDW) and superconducting (SC) correlations is essentially excluded from the model phase diagram.15.16 It is only when deviations from perfect electron-hole symmetry (nesting) are introduced and the long-range component of the SDW order is suppressed that superconductivity can be actually realized in place of magnetism ${ }^{17.18}$ In the anisotropic metallic phase where this suppression takes place, interchain Cooper pairing is enhanced and superconductivity emerges from the coupling between the weakened electron-hole and the still singular electron-electron scattering channels. Recent calculations ${ }^{19.20 .21}$ using the renormalization group (RG) method did confirm the existence of such an electronic pairing mechanism beyond the level of single-channel RPA-like approaches ${ }^{22.23 .24 .25 .26}$ A smooth crossover from the SDW state to superconductivity has then been found whenever the amplitude of nesting deviations reaches some threshold - a result in accordance with the sequence of transitions observed as a function of pressure ${ }^{9.11 .27}$

The RG approach shows nevertheless that whenever antiferromagnetism stands out as the dominant correlation in the normal state, the most stable interchain pairing is invariably a spin singlet state corresponding to a ' $d$-wave' symmetry gap with nodes on the Fermi surface. Although some experimental findings in the Bechgaard salts and their sulfur analogs do agree with this type of pairing ${ }_{, 2,28}$ other series of observations have rather been interpreted in support of a triplet order parameter, ${ }^{29.30 .31 .32}$ thus challenging the singlet scenario for superconductivity. Though there is too little so far to favor one scenario over another, these observations 
bring us to the question of whether triplet superconductivity can be possible or not when short-range antiferromagnetic correlations are dominant in the metallic state. As is well known for the electron gas model, there is a region of the phase diagram where triplet ' $p$-wave' superconductivity does exist as the most stable state. It has been suggested, on a phenomenological basis, that such a state is realized in the Bechgaard salts $\frac{33}{3}$ Within a microscopic approach, the region where $p$-wave superconductivity is stable is defined by irrelevant Umklapp scattering and by a backward scattering coupling that is much larger than forward scattering. However, this description pattern for superconductivity can be considered unsatisfactory given the unrealistic constraint it puts on the coupling constants, and for the suppression of both the antiferromagnetism at short distance and the Mott pseudo gap in the charge sector ${ }^{3}$ These flaws can hardly be reconciled with the related phenomenology of these molecular compounds observed around the critical pressure for superconductivity, when either the temperature or the magnetic field is varied,1.14.34.35.36

Another way to look at this problem is to consider more closely the effect of charge fluctuations on superconductivity. Staggered charge fluctuations are known to favor triplet pairing at odd but large angular momentum, a mechanism whose roots go back to the early work of Kohn and Luttinger about Cooper pairing in the presence of charge - Friedel - oscillations in isotropic

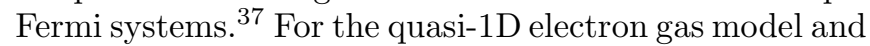
its version for lattice electrons, recent calculations have shown that triplet $f$-wave pairing is indeed enhanced when intrachain couplings are chosen to boost chargedensity-wave (CDW) fluctuations close to the level found in the SDW channel 21.38.39.40 However, for realistic repulsive couplings, $d$-wave pairing still remains tied to the highest critical temperature and hence to the most stable state for superconductivity.

All this goes to establish the robustness of $d$-wave pairing for the model with repulsive intrachain couplings and nesting deviations. It turns out, however, that the model is incomplete when charge fluctuations are found along the chains since then interchain Coulomb interaction is also present in practice. The inclusion of direct interchain electron-electron scattering processes, which will be denoted by $g_{i=1,2,3}^{\perp}$ in the following, defines the quasi-1D electron gas model in its extended form $\stackrel{41}{\underline{1}}$ At large momentum transfer, the interchain interaction is well known to favor a CDW ordered state $\stackrel{41,42,43,44.45}{ }$ This mechanism is mostly responsible for CDW long-range order observed in several organic and inorganic low-dimensional solids $\stackrel{46.47}{ }$ The physical relevance of interchain interactions in the Bechgaard salts, besides the intrachain $g_{i}$ and $t_{\perp}$, is supported by x-ray studies, which revealed that the SDW phase of these compounds is actually accompanied by CDW order ${ }^{48.49 .50}$ On the theoretical grounds, very little is known about the impact of adding direct interchain interactions on the structure of the phase diagram, especially in the repulsive sector when both a finite $t_{\perp}$ and nesting deviations are present.

In this work we wish to determine the possible densitywave and superconducting states of the extended quasi1D electron-gas model. In order to tackle this problem we shall apply the renormalization group method, which at the one-loop level has proved to be suited to reach a controlled description of interfering density-wave and superconducting channels of correlations. Among the results reported below, we have the unexpected finding that a small repulsive interchain backscattering term $g_{1}^{\perp}>0$ is sufficient to make $d$-wave superconductivity unstable to the benefit of a triplet $f$-wave phase. This occurs despite dominant SDW correlations in the metallic state and stable itinerant antiferromagnetism at lower nesting deviations. Under the latter conditions, SDW order becomes in turn unstable to the formation of a CDW state when the amplitude of $g_{1}^{\perp}$ exceeds some critical value. While these features persist when commensurability effects are taken into account and small - half-filling - Umklapp scattering is included, the effect of interchain forward scattering $\left(g_{2}^{\perp}>0\right)$ is found to frustrate nearestneighbor interchain $d$ - and $f$-wave pairing and to favor instead superconductivity with next-nearest-neighbor interchain pairing. Part of these results have been reported in Refs. 51,52 .

In Section II we introduce the model and the RG scheme employed for the four point vertices and the response functions. By way of illustration, the RG results at the one-loop level are given for purely intrachain interactions. In Section [II] we present the results for non zero interchain backward and forward interactions by which the different possibilities of ordered states in the phase diagram are obtained in the incommensurate case. The influence of Umklapp processes in the half-filled case is examined in Section IV A discussion of the results is given in section $\nabla$ where a possible connection between theory and experiments is made.

\section{THE EXTENDED ELECTRON GAS MODEL}

\section{A. Model}

We consider a lattice of $N_{\perp}$ coupled metallic chains described by the partition function $Z=$ $\iint D \psi^{*} D \psi e^{-\left(S_{0}+S_{I}\right)}$, which is expressed as a functional integration over anticommuting (Grassmann) fields $\psi$. Here $S_{0}$ and $S_{I}$ are the noninteracting and interacting parts of the action, respectively. The former part is given by

$$
S_{0}=-\sum_{K} \psi_{K}^{*}\left(i k_{0}-\xi_{\mathbf{k}}\right) \psi_{K}
$$

where $K=(\sigma, k), k=\left(k_{0} ; \mathbf{k}\right), k_{0}$ is the fermionic Matsubara frequency, $\mathbf{k}=\left(k_{\|}, k_{\perp}\right)$, the wave vector, and $\sigma= \pm$, the spin of the fermion field. The kinetic energy 
is given by

$$
\begin{aligned}
\xi_{\mathbf{k}} & =\epsilon_{\mathbf{k}}-\mu=v_{F}\left(\left|k_{\|}\right|-k_{F}\right)+\epsilon_{\perp}\left(k_{\perp}\right), \\
\epsilon_{\perp}\left(k_{\perp}\right) & =-2 t_{\perp} \cos k_{\perp}-2 t_{\perp}^{\prime} \cos 2 k_{\perp},
\end{aligned}
$$

where $\mu$ is the chemical potential taken as temperature independent. Here we have linearized the spectrum in the chain direction, using $v_{F}=2 t_{\|} \sin k_{F}$ as the longitudinal Fermi velocity, $k_{F}$ being the parallel Fermi wave vector if $t_{\perp}=0$. Throughout this work both the chain lattice constant $a$ and the interchain distance $d_{\perp}$ are put equal to unity. The interchain single electron hopping $t_{\perp}$ is considered small with respect to the longitudinal bandwidth $2 \Lambda_{0}$. In the following, we will take $2 \Lambda_{0}=30 t_{\perp}$, which is a typical figure for the anisotropy ratio in quasi$1 \mathrm{D}$ conductors like the Bechgaard and Fabre salts 53,54 The next nearest-neighbor hopping $t_{\perp}^{\prime} \ll t_{\perp}$ in the transverse direction gives the amplitude of nesting deviations. In Eq. (3), we have neglected the possibility of hopping in the third direction, which does not have any sizeable effect on our calculations. Its existence is of course crucial for the stabilization of true long-range order at finite temperature.

In the framework of the quasi-1D electron gas model,${ }^{6.16}$ the electron-electron interaction is parameterized by means of the g-ology approach. One first distinguishes between right and left moving fermions, depending on their velocity along the chains, so that the Grassmann variables $\psi_{K}^{(*)}$ become

$$
\psi_{K}^{(*)}= \begin{cases}R_{K}^{(*)} & \text { if } k_{\|}>0 \\ L_{K}^{(*)} & \text { if } k_{\|}<0 .\end{cases}
$$

Using these definitions, the interaction part of the action takes the form

$$
\begin{aligned}
S_{I}= & \frac{T}{N} \sum_{k_{1}^{\prime} k_{2}^{\prime} k_{2} k_{1}} \sum_{\sigma \sigma^{\prime}} \delta_{k_{1}^{\prime}+k_{2}^{\prime}, k_{2}+k_{1} \bmod G} \\
& \times\left\{g_{1}\left(k_{1}^{\prime} k_{2}^{\prime} k_{2} k_{1}\right) R_{k_{1}^{\prime} \sigma}^{*} L_{k_{2}^{\prime} \sigma^{\prime}}^{*} R_{k_{2} \sigma^{\prime}} L_{k_{1} \sigma}\right. \\
& +g_{2}\left(k_{1}^{\prime} k_{2}^{\prime} k_{2} k_{1}\right) R_{k_{1}^{\prime} \sigma}^{*} L_{k_{2}^{\prime} \sigma^{\prime}}^{*} L_{k_{2} \sigma^{\prime}} R_{k_{1} \sigma} \\
& \left.+\frac{1}{2}\left(g_{3}\left(k_{1}^{\prime} k_{2}^{\prime} k_{2} k_{1}\right) R_{k_{1}^{\prime} \sigma}^{*} R_{k_{2}^{\prime} \sigma^{\prime}}^{*} L_{k_{2} \sigma^{\prime}} L_{k_{1} \sigma}+\text { c.c. }\right)\right\},
\end{aligned}
$$

where $N$ is the number of lattice sites and $G=\left(0 ; 4 k_{F}, 0\right)$ the reciprocal lattice vector along the chains, which is involved in the Umklapp scattering at half-filling. Here we have omitted the so-called $g_{4}$ contribution for the interaction of electrons of the same branch since this coupling does not contribute to the singular channels of correlation in the renormalization group flow at the one-loop level. If we restrict ourselves to intrachain and nearestneighbor chain interactions, the amplitudes of the bare interactions are given by

$$
g_{j}\left(k_{\perp 1}^{\prime} k_{\perp 2}^{\prime} k_{\perp 2} k_{\perp 1}\right)=g_{j}+2 g_{j}^{\perp} \cos \left(k_{\perp 1}^{\prime}-k_{\perp 1}\right),
$$

where the only - transverse - momentum dependence comes from the interchain interaction. In this work we analyze the properties of the model for repulsive $g_{j}, g_{j}^{\perp}$ and finite Umklapp scattering, which is the physically relevant sector for real materials like the Bechgaard salts and their sulfur analogs.

\section{B. Renormalization group equations for the interactions}

The renormalization group can be applied to the problem of quasi-1D systems of interacting electrons in different ways 19.55 .56 .57 In the following, we shall adopt the so-called one-particle irreducible (1PI) momentum shell RG scheme, as developed by Honerkamp et al ${ }^{58}$ (cf. also Binz et al $\left.l^{59}\right)$. In this scheme, the 1PI vertex functions $\Gamma_{\Lambda}\left(K_{j}\right)$ of a physical system with infrared cutoff $\Lambda$ are calculated. Only degrees of freedom with energies equal to or greater than the cutoff are integrated out, which allows one to establish a direct link with the low energy effective action $S_{\Lambda}^{e f f}$ with an ultraviolet cutoff, as obtained in a Kadanoff-Wilson renormalization group scheme. According to Morris $\stackrel{60}{=}$ if $\left|\epsilon_{\mathbf{k}_{j}}\right|<\Lambda$, the effective interactions $g_{\Lambda}^{\text {eff }}\left(K_{j}\right)$ that appear in $S_{\Lambda}^{\text {eff }}$ are simply $g_{\Lambda}^{e f f}\left(K_{j}\right)=\Gamma_{\Lambda}\left(K_{j}\right)$.

The free particle propagator is suppressed for energies below an infrared cutoff $\Lambda$,

$$
C_{K}=\left\langle\psi_{K} \psi_{K}^{*}\right\rangle_{S_{0}}=\Theta\left(\left|\xi_{\mathbf{k}}\right|-\Lambda\right) \times \frac{-1}{i k_{0}-\xi_{\mathbf{k}}},
$$

where $\Theta$ is the step function. Setting all vertices involving more than two particles to zero, the renormalization group equations to the one-loop level are obtained. We also neglect self-energy corrections.

It is practically impossible to take into account the complete functional dependence of the vertices, which will in general vary with the wave vectors $\mathbf{k}$ as well as with the frequencies $k_{0}$ of the incoming and outgoing particles. By means of scaling arguments, one can show that the frequency dependence is irrelevant in the renormalization group sense, cf. for example Ref. 61. The same is true for the dependence on the distance of the wave vector $\mathbf{k}$ from the Fermi surface. Irrelevant variables are not necessarily negligible, in the sense that they may in principle have an influence on the RG flow before they vanish. However, the $\mathbf{k}_{s}$ dependence, with $\mathbf{k}_{s}$ the projection of the wave vector on the Fermi surface, remains by far the most important one in the low energy limit. We will hence only consider the dependence of the functions $\Gamma$ on the positions of the wave vectors along the Fermi surface, which we will parameterize by $k_{\perp}$. Due to momentum conservation, it is in general impossible to have all four arguments of a given vertex on the Fermi surface. Usually, the flow of the vertices with three arguments on the Fermi surface is calculated. In the case of imperfect nesting, such a procedure will underestimate the SDW correlations. In our case, the strong anisotropy of the dispersion relation allows us to make a slightly different 
choice. We fix the values of $k_{\|}$and $k_{0}$ such that the most divergent contributions to the renormalization group flow are taken into account. If we define the Cooper, Landau, and Peierls momentum variables

$$
\begin{aligned}
& q_{\mathcal{C}}=k_{1}+k_{2}, \\
& q_{\mathcal{L}}= \begin{cases}k_{1}^{\prime}-k_{2} & \text { for } \Gamma_{1}, \\
k_{1}^{\prime}-k_{1} & \text { for } \Gamma_{2},\end{cases} \\
& q_{\mathcal{P}}= \begin{cases}k_{1}^{\prime}-k_{1} & \text { for } \Gamma_{1}, \\
k_{1}^{\prime}-k_{2} & \text { for } \Gamma_{2} \text { and } \Gamma_{3},\end{cases} \\
& q_{\mathcal{P}}^{\prime}=k_{1}^{\prime}-k_{1} \text { for } \Gamma_{3},
\end{aligned}
$$

we can write the one-loop RG equations in the form

$$
\begin{aligned}
\frac{d}{d l} \Gamma\left(q_{\mathcal{C}}, q_{\mathcal{L}}, q_{\mathcal{P}}\right)= & \sum_{k_{\text {loop }}} \operatorname{Cooper}\left(\Gamma\left(q_{\mathcal{C}}, \tilde{q}_{\mathcal{L}}, \tilde{q}_{\mathcal{P}}\right)\right) \\
& +\sum_{k_{\text {loop }}} \operatorname{Peierls}\left(\Gamma\left(\tilde{q}_{\mathcal{C}}, \tilde{q}_{\mathcal{L}}, q_{\mathcal{P}}\right)\right)
\end{aligned}
$$

In this equation, $\tilde{q}_{\mathcal{C}}, \tilde{q}_{\mathcal{L}}$ and $\tilde{q}_{\mathcal{P}}$ are functions of the internal loop momentum-frequency $k_{\text {loop}}$. The most divergent contributions to the RG flow are obtained if $q_{\mathcal{C}, 0}=q_{\mathcal{P}, 0}^{(\prime)}=0$ for the frequencies, as well as

$$
\begin{aligned}
q_{\mathcal{C} \|} & =0, q_{\mathcal{P} \|}=2 k_{F} \text { for } \Gamma_{1,2}, \\
q_{\mathcal{P} \|}^{(\prime)} & = \pm 2 k_{F} \text { for } \Gamma_{3},
\end{aligned}
$$

for the longitudinal components. Note that, for either choice of the $k_{\|}$dependence, one does not obtain a closed set of equations. The renormalization group equations for vertices for which relations (9) are valid contain vertex functions with different values of $q_{\mathcal{C}}, q_{\mathcal{P}}$. We will therefore need to replace these functions by those calculated by imposing conditions (9).

For the dimensionless vertices $\tilde{\Gamma}=\Gamma / \pi v_{F}$, we thus obtain the equations 62

$$
\begin{aligned}
& \dot{\tilde{\Gamma}}_{1}\left(k_{\perp 1}^{\prime} k_{\perp 2}^{\prime} k_{\perp 2} k_{\perp 1}\right)=-\frac{1}{N_{\perp}} \sum_{k_{\perp}}\left[B_{\mathcal{C}}\left(k_{\perp}, q_{\mathcal{C} \perp}\right)\right. \\
& \times\left\{\tilde{\Gamma}_{1}\left(k_{\perp 1}^{\prime} k_{\perp 2}^{\prime} k_{\perp} k_{\perp}^{\prime}\right) \tilde{\Gamma}_{2}\left(k_{\perp} k_{\perp}^{\prime} k_{\perp 1} k_{\perp 2}\right)\right. \\
& \left.\left.+\tilde{\Gamma}_{2}\left(k_{\perp 1}^{\prime} k_{\perp 2}^{\prime} k_{\perp}^{\prime} k_{\perp}\right) \tilde{\Gamma}_{1}\left(k_{\perp} k_{\perp}^{\prime} k_{\perp 2} k_{\perp 1}\right)\right\}\right]\left.\right|_{\substack{k_{\perp}^{\prime}=-k_{\perp}+q_{\mathcal{C} \perp} \\
q_{\mathcal{C} \perp}=k_{\perp 1}+k_{\perp 2}}} \\
& +\frac{1}{N_{\perp}} \sum_{k_{\perp}}\left[B_{\mathcal{P}}\left(k_{\perp}, q_{\mathcal{P} \perp}\right)\right. \\
& \times\left\{\left(\tilde{\Gamma}_{2}\left(k_{\perp 1}^{\prime} k_{\perp} k_{\perp 1} k_{\perp}^{\prime}\right)-\tilde{\Gamma}_{1}\left(k_{\perp 1}^{\prime} k_{\perp} k_{\perp}^{\prime} k_{\perp 1}\right)\right) \tilde{\Gamma}_{1}\left(k_{\perp}^{\prime} k_{\perp 2}^{\prime} k_{\perp 2} k_{\perp}\right)\right. \\
& +\tilde{\Gamma}_{1}\left(k_{\perp 1}^{\prime} k_{\perp} k_{\perp}^{\prime} k_{\perp 1}\right)\left(\tilde{\Gamma}_{2}\left(k_{\perp}^{\prime} k_{\perp 2}^{\prime} k_{\perp} k_{\perp 2}\right)-\tilde{\Gamma}_{1}\left(k_{\perp}^{\prime} k_{\perp 2}^{\prime} k_{\perp 2} k_{\perp}\right)\right) \\
& +\left(\tilde{\Gamma}_{3}\left(k_{\perp 1}^{\prime} k_{\perp} k_{\perp 1} k_{\perp}^{\prime}\right)-\tilde{\Gamma}_{3}\left(k_{\perp 1}^{\prime} k_{\perp} k_{\perp}^{\prime} k_{\perp 1}\right)\right) \tilde{\Gamma}_{3}\left(k_{\perp}^{\prime} k_{\perp 2}^{\prime} k_{\perp 2} k_{\perp}\right) \\
& \left.\left.+\tilde{\Gamma}_{3}\left(k_{\perp 1}^{\prime} k_{\perp} k_{\perp}^{\prime} k_{\perp 1}\right)\left(\tilde{\Gamma}_{3}\left(k_{\perp}^{\prime} k_{\perp 2}^{\prime} k_{\perp} k_{\perp 2}\right)-\tilde{\Gamma}_{3}\left(k_{\perp}^{\prime} k_{\perp 2}^{\prime} k_{\perp 2} k_{\perp}\right)\right)\right\}\right]\left.\right|_{\substack{k_{\perp}^{\prime}=k_{\perp}+q_{\mathcal{P} \perp} \\
q_{\mathcal{P}}=k_{\perp 1}^{\prime}-k_{\perp 1}}}, \\
& \dot{\tilde{\Gamma}}_{2}\left(k_{\perp 1}^{\prime} k_{\perp 2}^{\prime} k_{\perp 2} k_{\perp 1}\right)=-\frac{1}{N_{\perp}} \sum_{k_{\perp}}\left[B_{\mathcal{C}}\left(k_{\perp}, q_{\mathcal{C} \perp}\right)\right. \\
& \times\left\{\tilde{\Gamma}_{1}\left(k_{\perp 1}^{\prime} k_{\perp 2}^{\prime} k_{\perp} k_{\perp}^{\prime}\right) \tilde{\Gamma}_{1}\left(k_{\perp} k_{\perp}^{\prime} k_{\perp 1} k_{\perp 2}\right)\right. \\
& \left.\left.+\tilde{\Gamma}_{2}\left(k_{\perp 1}^{\prime} k_{\perp 2}^{\prime} k_{\perp}^{\prime} k_{\perp}\right) \tilde{\Gamma}_{2}\left(k_{\perp} k_{\perp}^{\prime} k_{\perp 2} k_{\perp 1}\right)\right\}\right]\left.\right|_{\begin{array}{c}
k_{\perp}^{\prime}=-k_{\perp}+q_{\mathcal{C} \perp} \\
q_{\mathcal{C} \perp}=k_{\perp 1}+k_{\perp 2}
\end{array}} \\
& +\frac{1}{N_{\perp}} \sum_{k_{\perp}}\left[B_{\mathcal{P}}\left(k_{\perp}, q_{\mathcal{P} \perp}\right)\right. \\
& \times\left\{\tilde{\Gamma}_{2}\left(k_{\perp 1}^{\prime} k_{\perp} k_{\perp 2} k_{\perp}^{\prime}\right) \tilde{\Gamma}_{2}\left(k_{\perp}^{\prime} k_{\perp 2}^{\prime} k_{\perp} k_{\perp 1}\right)\right. \\
& \left.\left.+\tilde{\Gamma}_{3}\left(k_{\perp 1}^{\prime} k_{\perp} k_{\perp 2} k_{\perp}^{\prime}\right) \tilde{\Gamma}_{3}\left(k_{\perp}^{\prime} k_{\perp 2}^{\prime} k_{\perp} k_{\perp 1}\right)\right\}\right]\left.\right|_{\substack{k_{\perp}^{\prime}=k_{\perp}+q_{\mathcal{P} \perp} \\
q_{\mathcal{P} \perp}=k_{\perp 1}^{\prime}-k_{\perp 2}}},
\end{aligned}
$$




$$
\begin{aligned}
& \dot{\tilde{\Gamma}}_{3}\left(k_{\perp 1}^{\prime} k_{\perp 2}^{\prime} k_{\perp 2} k_{\perp 1}\right)=\frac{1}{N_{\perp}} \sum_{k_{\perp}}\left[B_{\mathcal{P}}\left(k_{\perp}, q_{\mathcal{P} \perp}^{\prime}\right)\right. \\
& \times\left\{\left(\tilde{\Gamma}_{2}\left(k_{\perp 1}^{\prime} k_{\perp} k_{\perp 1} k_{\perp}^{\prime}\right)-\tilde{\Gamma}_{1}\left(k_{\perp 1}^{\prime} k_{\perp} k_{\perp}^{\prime} k_{\perp 1}\right)\right) \tilde{\Gamma}_{3}\left(k_{\perp}^{\prime} k_{\perp 2}^{\prime} k_{\perp 2} k_{\perp}\right)\right. \\
& +\tilde{\Gamma}_{1}\left(k_{\perp 1}^{\prime} k_{\perp} k_{\perp}^{\prime} k_{\perp 1}\right)\left(\tilde{\Gamma}_{3}\left(k_{\perp}^{\prime} k_{\perp 2}^{\prime} k_{\perp} k_{\perp 2}\right)-\tilde{\Gamma}_{3}\left(k_{\perp}^{\prime} k_{\perp 2}^{\prime} k_{\perp 2} k_{\perp}\right)\right) \\
& +\left(\tilde{\Gamma}_{3}\left(k_{\perp 1}^{\prime} k_{\perp} k_{\perp 1} k_{\perp}^{\prime}\right)-\tilde{\Gamma}_{3}\left(k_{\perp 1}^{\prime} k_{\perp} k_{\perp}^{\prime} k_{\perp 1}\right)\right) \tilde{\Gamma}_{1}\left(k_{\perp}^{\prime} k_{\perp 2}^{\prime} k_{\perp 2} k_{\perp}\right) \\
& \left.\left.+\tilde{\Gamma}_{3}\left(k_{\perp 1}^{\prime} k_{\perp} k_{\perp}^{\prime} k_{\perp 1}\right)\left(\tilde{\Gamma}_{2}\left(k_{\perp}^{\prime} k_{\perp 2}^{\prime} k_{\perp} k_{\perp 2}\right)-\tilde{\Gamma}_{1}\left(k_{\perp}^{\prime} k_{\perp 2}^{\prime} k_{\perp 2} k_{\perp}\right)\right)\right\}\right]\left.\right|_{\substack{k_{\perp}^{\prime}=k_{\perp}+q_{\mathcal{P}}^{\prime} \\
q_{\mathcal{P} \perp}^{\prime}=k_{\perp 1}^{\prime}-k_{\perp 1}}} \\
& +\frac{1}{N_{\perp}} \sum_{k_{\perp}}\left[B_{\mathcal{P}}\left(k_{\perp}, q_{\mathcal{P} \perp}\right)\right. \\
& \times\left\{\tilde{\Gamma}_{2}\left(k_{\perp 1}^{\prime} k_{\perp} k_{\perp 2} k_{\perp}^{\prime}\right) \tilde{\Gamma}_{3}\left(k_{\perp}^{\prime} k_{\perp 2}^{\prime} k_{\perp} k_{\perp 1}\right)\right. \\
& \left.\left.+\tilde{\Gamma}_{3}\left(k_{\perp 1}^{\prime} k_{\perp} k_{\perp 2} k_{\perp}^{\prime}\right) \tilde{\Gamma}_{2}\left(k_{\perp}^{\prime} k_{\perp 2}^{\prime} k_{\perp} k_{\perp 1}\right)\right\}\right]\left.\right|_{\substack{k_{\perp}^{\prime}=k_{\perp}+q_{\mathcal{P} \perp} \\
q_{\mathcal{P} \perp=k_{\perp 1}^{\prime}-k_{\perp 2}}}},
\end{aligned}
$$

where the dot denotes derivation with respect to $-\ln \left(\Lambda / \Lambda_{0}\right)$. The particle-particle $(\mathcal{C})$ and particle-hole $(\mathcal{P})$ loops, after summation over Matsubara frequencies and longitudinal wave vectors, are given by

$$
\begin{aligned}
B_{\mathcal{C} / \mathcal{P}}\left(k_{\perp}, q_{\perp}\right)= & \sum_{\nu= \pm 1} \Theta\left(\left|\Lambda+\nu A_{\mathcal{C} / \mathcal{P}}\right|-\Lambda\right) \\
& \times \frac{1}{2}\left(\tanh \frac{\Lambda+\nu A_{\mathcal{C} / \mathcal{P}}}{2 T}+\tanh \frac{\Lambda}{2 T}\right) \\
& \times \frac{\Lambda}{2 \Lambda+\nu A_{\mathcal{C} / \mathcal{P}}}, \\
A_{\mathcal{C}}\left(k_{\perp}, q_{\perp}\right) & =-\epsilon_{\perp}\left(k_{\perp}\right)+\epsilon_{\perp}\left(-k_{\perp}+q_{\perp}\right), \\
A_{\mathcal{P}}\left(k_{\perp}, q_{\perp}\right) & =-\epsilon_{\perp}\left(k_{\perp}\right)-\epsilon_{\perp}\left(k_{\perp}+q_{\perp}\right) .
\end{aligned}
$$

For continuity reasons, we always take $\Theta(0):=\frac{1}{2}$. The starting values are given by the bare interactions, cf. section IIA

It is useful to consider the following limiting cases for the RG equations. As long as $\Lambda \gg t_{\perp}$, we may take their 1D limit $\left(t_{\perp}=0\right)$. In this regime, the Cooper and Peierls renormalization channels are entirely coupled, in the sense that all vertices are strongly renormalized in both channels (except for Umklapp processes, which only appear in the Peierls channel). When $\Lambda \ll t_{\perp}$, the coupling between the Cooper and Peierls channels is weak in the sense that, depending on the arguments $k_{\perp}$, most vertices are strongly renormalized in only one (or no) channel at a time. Nevertheless, the remaining interplay between the channels is at the origin of spinand charge-fluctuation-induced superconductivity in the weak coupling regime. For purely repulsive interactions, the Peierls channel is the most important one as long as deviations from perfect nesting may be neglected, $i$. e. for $\Lambda \gg t_{\perp}^{\prime}$. In the presence of attractive effective interactions, the Cooper channel plays an important role, and will be dominant when $\Lambda \ll t_{\perp}^{\prime}$. The RG equations are written in these limits in appendix $\mathrm{A}$.

A similar dimensional crossover may be observed with respect to the temperature instead of $\Lambda$. As long as $T \gg t_{\perp}$, the functions we calculate do not vary very strongly with the transverse wave vector, contrary to the low temperature case. An example is given in Fig. 2(a).

\section{Response functions}

In order to evaluate susceptibilities, we add a term of the form

$$
\begin{aligned}
S_{h}= & \sum_{\alpha} \sum_{q} h_{\alpha S C}^{*}(q) O_{\alpha S C}(q)+\text { c.c. } \\
& +\sum_{\alpha, M} \sum_{q} h_{\alpha D W}^{(M) *}(q) O_{\alpha D W}^{(M)}(q)+\text { c.c. }
\end{aligned}
$$

to the action. The first term describes pairing and the second term density-wave correlations. The external fields $h^{(*)}(q)$ are taken to be infinitesimal. They couple to pairs of fermionic variables which we will define in the following. Let us first introduce the particle-particle operators

$$
o_{\alpha}(k, q)=\sum_{\sigma^{\prime} \sigma} \tau_{\sigma^{\prime} \sigma}^{(\alpha)} L_{-k+q, \sigma^{\prime}} R_{k, \sigma}
$$

for singlet $(\alpha=s)$ and triplet $\left(\alpha=t_{x, y, z}\right)$ pairs. The spin dependence is given by the coefficients

$$
\begin{aligned}
& \tau_{\sigma^{\prime} \sigma}^{(s)}=\sigma \delta_{\sigma^{\prime},-\sigma}, \\
& \tau_{\sigma^{\prime} \sigma}^{\left(t_{x}\right)}=-\sigma \delta_{\sigma^{\prime} \sigma}, \tau_{\sigma^{\prime} \sigma}^{\left(t_{y}\right)}=-i \delta_{\sigma^{\prime} \sigma}, \tau_{\sigma^{\prime} \sigma}^{\left(t_{z}\right)}=\delta_{\sigma^{\prime},-\sigma}
\end{aligned}
$$

$(\sigma= \pm 1)$. The pair operator appearing in $S_{h}$ is defined as

$$
O_{\alpha S C}(q)=\sqrt{\frac{T}{N}} \sum_{\left\{k ; k_{\|}>0\right\}} z_{\alpha}(q-k, k) o_{\alpha}(k, q) .
$$




\begin{tabular}{|l|l|l|}
\hline name & spin pairing & $\Delta_{r}\left(k_{\perp}\right)$ \\
\hline \hline$s$ & singlet & 1 \\
\hline$p_{x}$ & triplet & $r$ \\
$p_{y}$ & & $\sin k_{\perp}$ \\
\hline$d_{x^{2}-y^{2}}$ & singlet & $\begin{array}{l}\cos k_{\perp} \\
d_{x y} \sin k_{\perp}\end{array}$ \\
\hline$f$ & triplet & $\begin{array}{l}r \cos k_{\perp} \\
\sin 2 k_{\perp}\end{array}$ \\
\hline$g$ & singlet & $\begin{array}{l}\cos 2 k_{\perp} \\
r \sin 2 k_{\perp}\end{array}$ \\
\hline$h$ & triplet & $r \cos 2 k_{\perp}$ \\
\hline$i$ & singlet & $\cos 3 k_{\perp}$ \\
\hline
\end{tabular}

TABLE I: Superconducting order parameters in a quasi-1D geometry. The names are assigned according to the number of sign changes along the Fermi surface.

The function $z_{\alpha}$ describes the orbital symmetry of the superconducting order parameter. We classify these order parameters by their behavior on the Fermi surface. They are parameterized by $k_{\perp}$ and $r=\operatorname{sgn} k_{\|}$. A list of the superconducting order parameters we shall examine is given in Table With these, we have

$$
z_{\alpha}\left(k_{\perp}^{\prime}, k_{\perp}\right)= \begin{cases}1 & \text { for } s \text { and } p_{x} \\ \sqrt{2} \Delta_{r=+}\left(k_{\perp}\right) & \text { for all others }\end{cases}
$$

Similarly, we introduce particle-hole operators

$$
o_{\alpha}(k, q)=\sum_{\sigma^{\prime} \sigma} \sigma_{\sigma^{\prime} \sigma}^{(\alpha)} L_{k-q, \sigma^{\prime}}^{*} R_{k, \sigma}
$$

for charge $(\alpha=C)$ and $\operatorname{spin}\left(\alpha=S_{x, y, z}\right)$ excitations. Here, $\sigma^{(C)}$ is the $2 \times 2$ identity matrix, and $\sigma^{\left(S_{x, y, z}\right)}$ are the Pauli matrices. At half filling, one has to distinguish between bond and site density waves. In direct space, these are given by

$O_{x}= \begin{cases}\frac{1}{2} \sum_{\sigma^{\prime} \sigma} \sigma_{\sigma^{\prime} \sigma}^{(\alpha)} \psi_{x, \sigma^{\prime}}^{*} \psi_{x, \sigma} & \text { for site DW, } \\ \frac{1}{4} \sum_{\sigma^{\prime} \sigma}\left(\sigma_{\sigma^{\prime} \sigma}^{(\alpha)} \psi_{x, \sigma^{\prime}}^{*} \psi_{x+d, \sigma}+\text { c.c. }\right) & \text { for bond DW },\end{cases}$

where $d=\left(0 ; d_{\|}, 0\right)$, and $d_{\|}$is the lattice periodicity along the chains. Since we have $k_{F} d_{\|}=\frac{\pi}{2}$, the associated Fourier transforms are, for $q_{\|} \approx 2 k_{F}$,

$$
O_{\alpha D W}^{(M)}(q) \approx \frac{1}{2} \sqrt{\frac{T}{N}} \sum_{\left\{k ; k_{\|}>0\right\}}\left[z_{\alpha}^{(M)}(k-q, k) o_{\alpha}(k, q)+M\left(z_{\alpha}^{(M)}(k+q-G, k)\right)^{*} o_{\alpha}^{*}(k, G-q)\right]
$$

with $M=+$ for site and $M=-$ for bond density waves, and $z_{\alpha}^{(M)}(k, q)=1$. (For the case $M=-1$, we have neglected a constant imaginary factor.) Note that $\left(O_{\alpha}^{(M)}\right)^{*}=M O_{\alpha}^{(M)}$, and that $\left\langle O_{\alpha}^{(M)} O_{\alpha}^{(M)}\right\rangle$ and $\left\langle O_{\alpha}^{(M) *} O_{\alpha}^{(M) *}\right\rangle$ do not vanish, but contribute to the associated susceptibilities. Away from half filling, we only consider site density waves. In this case, only the first term in Eq. (22) contributes.

In the presence of $S_{h}$, the one-particle vertex contains a nondiagonal part with a contribution linear in $\left(h_{\alpha D W}^{(M)}(q)\right)^{*}$ of the form

$$
\sigma_{\sigma^{\prime} \sigma}^{(\alpha)} \delta_{k^{\prime}, k-q} \zeta_{\alpha}^{(M)}\left(k^{\prime}, k\right)
$$

(if $k_{\|}>0$ ). Also due to $S_{h}$, vertices with two outgoing, but no incoming, particles are now non-zero. Their linear part in $\left(h_{\alpha S C}(q)\right)^{*}$ takes the form

$$
\tau_{\sigma^{\prime} \sigma}^{(\alpha)} \delta_{k^{\prime}, q-k} \zeta_{\alpha}\left(k^{\prime}, k\right)
$$

(if $k_{\|}>0$ ). The vertex parts $\zeta_{\alpha}$ determine the renormalization of the susceptibilities $\chi_{\alpha}$ as follows. In the beginning of the flow, we have $\zeta_{\alpha}\left(k^{\prime}, k\right)=z_{\alpha}\left(k^{\prime}, k\right)$ and $\chi_{\alpha}=0$. The RG equations for density waves $(\alpha=C, S)$ are: 


$$
\begin{aligned}
\dot{\zeta}_{\alpha}^{M}\left(p_{\perp}, p_{\perp}+q_{\perp}\right)= & \frac{1}{N_{\perp}} \sum_{k_{\perp}} B_{\mathcal{P}}\left(k_{\perp}, q_{\perp}\right) \zeta_{\alpha}^{M}\left(k_{\perp}, k_{\perp}+q_{\perp}\right) \\
& \times\left[\tilde{\Gamma}_{\alpha}\left(k_{\perp}+q_{\perp}, p_{\perp}, k_{\perp}, p_{\perp}+q_{\perp}\right)\right. \\
& \left.+\delta_{q_{\|}, 2 k_{F}^{1 D}}\left(\delta_{q_{\perp}, 0}+\delta_{q_{\perp}, \pi / b}\right) M \tilde{\Gamma}_{3}^{(\alpha)}\left(k_{\perp}, p_{\perp}, k_{\perp}+q_{\perp}, p_{\perp}+q_{\perp}\right)\right], \\
\dot{\tilde{\chi}}_{\alpha}^{M}\left(q_{\perp}\right)= & -\frac{2}{N_{\perp}} \sum_{k_{\perp}} B_{\mathcal{P}}\left(k_{\perp}, q_{\perp}\right)\left|\zeta_{\alpha}^{M}\left(k_{\perp}, k_{\perp}+q_{\perp}\right)\right|^{2} .
\end{aligned}
$$

For Cooper pairs $(\alpha=s, t)$, we have:

$$
\begin{aligned}
\dot{\zeta}_{\alpha}\left(-p_{\perp}+q_{\perp}, p_{\perp}\right)= & \frac{1}{N_{\perp}} \sum_{k_{\perp}} B_{\mathcal{C}}\left(k_{\perp}, q_{\perp}\right) \zeta_{\alpha}\left(-k_{\perp}+q_{\perp}, k_{\perp}\right) \\
& \times \tilde{\Gamma}_{\alpha}\left(k_{\perp},-k_{\perp}+q_{\perp},-p_{\perp}+q_{\perp}, p_{\perp}\right), \\
\dot{\tilde{\chi}}_{\alpha}\left(q_{\perp}\right)= & -\frac{2}{N_{\perp}} \sum_{k_{\perp}} B_{\mathcal{C}}\left(k_{\perp}, q_{\perp}\right)\left|\zeta_{\alpha}\left(-k_{\perp}+q_{\perp}, k_{\perp}\right)\right|^{2} .
\end{aligned}
$$

$\tilde{\chi}$ is defined as $\pi v_{F \|} \chi$. We have introduced the linear combinations of vertex functions

$$
\begin{aligned}
\Gamma_{C}\left(k_{\perp 1}^{\prime}, k_{\perp 2}^{\prime}, k_{\perp 2}, k_{\perp 1}\right) & =-2 \Gamma_{1}\left(k_{\perp 1}^{\prime}, k_{\perp 2}^{\prime}, k_{\perp 1}, k_{\perp 2}\right)+\Gamma_{2}\left(k_{\perp 1}^{\prime}, k_{\perp 2}^{\prime}, k_{\perp 2}, k_{\perp 1}\right), \\
\Gamma_{S}\left(k_{\perp 1}^{\prime}, k_{\perp 2}^{\prime}, k_{\perp 2}, k_{\perp 1}\right) & =\Gamma_{2}\left(k_{\perp 1}^{\prime}, k_{\perp 2}^{\prime}, k_{\perp 2}, k_{\perp 1}\right), \\
\Gamma_{3}^{(C)}\left(k_{\perp 1}^{\prime}, k_{\perp 2}^{\prime}, k_{\perp 2}, k_{\perp 1}\right) & =-2 \Gamma_{3}\left(k_{\perp 1}^{\prime}, k_{\perp 2}^{\prime}, k_{\perp 1}, k_{\perp 2}\right)+\Gamma_{3}\left(k_{\perp 1}^{\prime}, k_{\perp 2}^{\prime}, k_{\perp 2}, k_{\perp 1}\right) \\
\Gamma_{3}^{(S)}\left(k_{\perp 1}^{\prime}, k_{\perp 2}^{\prime}, k_{\perp 2}, k_{\perp 1}\right) & =\Gamma_{3}\left(k_{\perp 1}^{\prime}, k_{\perp 2}^{\prime}, k_{\perp 2}, k_{\perp 1}\right) \\
\Gamma_{s}\left(k_{\perp 1}^{\prime}, k_{\perp 2}^{\prime}, k_{\perp 2}, k_{\perp 1}\right) & =-\Gamma_{1}\left(k_{\perp 1}^{\prime}, k_{\perp 2}^{\prime}, k_{\perp 1}, k_{\perp 2}\right)-\Gamma_{2}\left(k_{\perp 1}^{\prime}, k_{\perp 2}^{\prime}, k_{\perp 2}, k_{\perp 1}\right), \\
\Gamma_{t}\left(k_{\perp 1}^{\prime}, k_{\perp 2}^{\prime}, k_{\perp 2}, k_{\perp 1}\right) & =\Gamma_{1}\left(k_{\perp 1}^{\prime}, k_{\perp 2}^{\prime}, k_{\perp 1}, k_{\perp 2}\right)-\Gamma_{2}\left(k_{\perp 1}^{\prime}, k_{\perp 2}^{\prime}, k_{\perp 2}, k_{\perp 1}\right) .
\end{aligned}
$$

Interactions $g_{\alpha}\left(\alpha=C, S_{x, y, z}, s, t_{x, y, z}\right)$ and $g_{3}^{(C)}, g_{3}^{(S)}$ are defined analogously. Using these functions as well as the particle-particle and particle-hole pair operators defined above, and neglecting Umklapp processes, we can rewrite the interaction part of the action in the following way:

$$
\begin{aligned}
S_{I} & =-\frac{1}{2} \sum_{\alpha=C, S_{x, y, z}} \frac{T}{N} \sum_{k^{\prime} k q} g_{\alpha}\left(k^{\prime}, k-q, k^{\prime}-q, k\right) o_{\alpha}^{*}\left(k^{\prime}, q\right) o_{\alpha}(k, q) \\
& =-\frac{1}{2} \sum_{\alpha=s, t_{x, y, z}} \frac{T}{N} \sum_{k^{\prime} k q} g_{\alpha}\left(k^{\prime}, q-k^{\prime}, q-k, k\right) o_{\alpha}^{*}\left(k^{\prime}, q\right) o_{\alpha}(k, q),
\end{aligned}
$$

$g_{s, t}$ (resp. $g_{C, S}$ ) thus describes the interaction between particles forming a singlet or triplet pair (resp. particlehole pair). The relation between these couplings is as follows:

$$
\begin{aligned}
& g_{s}=\frac{1}{2}\left(3 g_{S}-g_{C}\right), \\
& g_{t}=\frac{1}{2}\left(g_{S}+g_{C}\right) .
\end{aligned}
$$

We will make use of these relations in the following, when we discuss density fluctuation induced Cooper pairing. Note that Umklapp processes do not couple to the Cooper channel, and therefore affect Cooper pair formation only indirectly via their effect on $g_{C}, g_{S}$.

The RG equations are solved numerically, using a fourth order Runge-Kutta algorithm ${ }^{63}$ with fixed step sizes (taking large steps in the beginning of the flow and short steps close to the divergence). The graphs showing transition temperatures are obtained with the help of an adaptive stepsize algorithm ${ }^{63.64}$ As far as the $k_{\perp}$ dependence of the vertex functions is concerned, we discretize the Fermi surface using 32 patches for each sheet. Taking advantage of all symmetries of the problem, we thus have to calculate 9010 different function values for each $\Gamma_{j}$. The use of twice the number of patches does not significantly modify our results. The integral of the functions $B_{\mathcal{C}, \mathcal{P}}$ must be calculated more precisely; we use a fourth order Runge-Kutta algorithm with adaptive step size. 


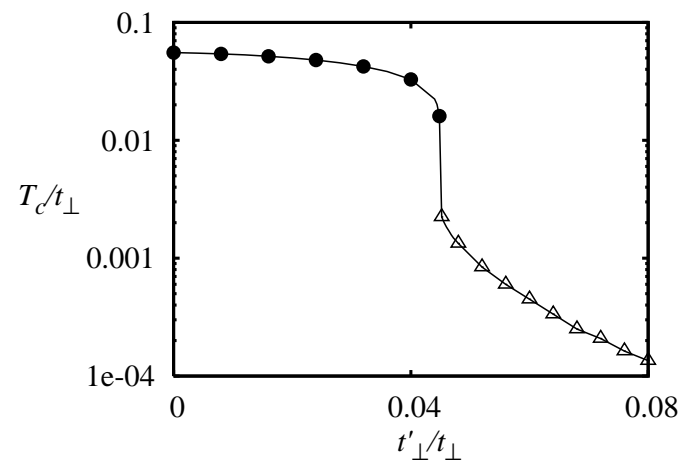

FIG. 1: RG phase diagram for the quasi-1D electron gas model at incommensurate filling $\left(g_{3}=0\right)$ with intrachain interactions $\left(g_{j}^{\perp}=0\right)$ and nesting deviations, parameterized by $t_{\perp}^{\prime}$. The circles indicate the transition temperature for SDW and the triangles that for SCd. All figures in this article are obtained using the bare intrachain interactions $\tilde{g}_{1}=0.32$, $\tilde{g}_{2}=0.64$ and the anisotropy ratio $\Lambda_{0} / t_{\perp}=15$.

\section{Results for the case of intrachain interactions}

To illustrate our method, we shall first consider the by now well known case of a quasi-1D electron gas model with purely intrachain interactions and no Umklapp scattering. This case has been studied in detail by Duprat and Bourbonnais $\frac{19}{9}$ using a Kadanoff-Wilson RG scheme and an approximation where only two independent momentum variables for the vertices were taken into account. We here use the 1PI scheme and retain the full three variable dependence for the vertices. Our results confirm those of reference 19 .

The RG calculations that follow are performed for the values $\tilde{g}_{1}=0.32$ and $\tilde{g}_{2}=0.64$, which are representative of the couplings likely to be found in practice in low dimensional conductors. Thus for not too large nesting deviations, the renormalization group flow scales to strong coupling, which leads to a singular behavior in the susceptibility of a particular channel of correlations. This signals an instability of the normal state towards an ordered phase. The phase diagram obtained as a function of $t_{\perp}^{\prime}$ is shown in Fig. 11 For good nesting, there is an SDW phase with a modulation wave vector $\mathbf{Q}_{0}=\left(2 k_{F}, \pi\right)$ that corresponds to the best nesting vector of the spectrum (2). The transition temperature obtained for perfect nesting $\left(t_{\perp}^{\prime}=0\right)$ is $T_{c}^{0} \approx 0.055 t_{\perp}$. If we take $t_{\perp} \approx 200 \mathrm{~K}$, we have $T_{c}^{0} \approx 11 \mathrm{~K}$, which falls in the range of the experimental $T_{c}$ for systems like the Bechgaard salts at low pressure. By increasing $t_{\perp}^{\prime}$, the transition temperature decreases until the threshold value $t_{\perp c}^{\prime} \approx 0.045 t_{\perp} \approx 9 \mathrm{~K}$ is reached, where the SDW is suppressed and replaced by $d$-wave superconductivity $(\mathrm{SC} d)$. The maximum temperature for the $\mathrm{SC} d$ state is found to be $T_{c}^{0}(\mathrm{SC} d) \approx 0.002 t_{\perp} \approx 0.4 \mathrm{~K}$. These estimates for $t^{\prime}$ and $T_{c}$ are comparable to the experimental results $\frac{9,19,65.66}{10}$
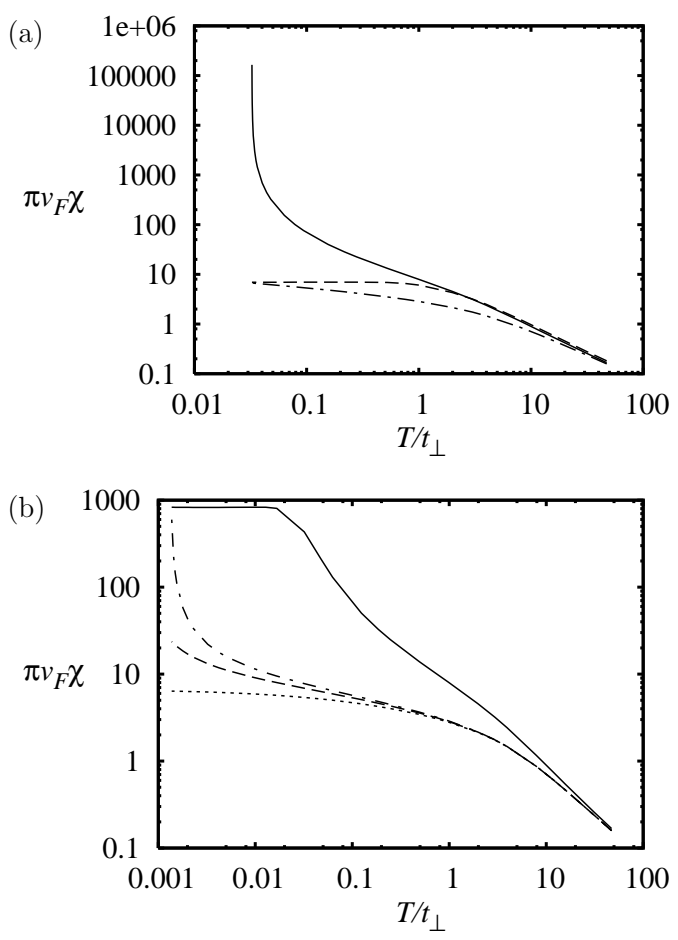

FIG. 2: Temperature dependence of the susceptibilities in the normal phase $\left(g_{3}=0, g_{j}^{\perp}=0\right)$ :

(a) above the SDW phase $\left(t_{\perp}^{\prime}=0.04 t_{\perp}\right)$. The continuous (dashed) line corresponds to the SDW response at $q_{\perp}=\pi(0)$; the dashed-dotted line to $\mathrm{SC} d$ correlations. It is interesting to observe that the temperature scale $T \sim t_{\perp}$ below which the $q_{\perp}=0$ curve separates and levels off corresponds to the so-called single particle dimensionality crossover. ${ }^{1}$ Our RG scheme thus captures this effect correctly.

(b) above the SC phase $\left(t_{\perp}^{\prime}=0.048 t_{\perp}\right)$. The continuous line corresponds to the SDW response with transverse modulation $\pi$, the dashed-dotted line to $\mathrm{SC}_{x^{2}-y^{2}}\left(\cos k_{\perp}\right)$, the dotted line to $\mathrm{SC}_{x y}\left(r \sin k_{\perp}\right)$ and the dashed line to $\mathrm{SC} g$ $\left(r \sin 2 k_{\perp}\right)$ correlations.

The order parameter of the low temperature phase can be identified in two different ways, which give equivalent results. The first one follows from the identification of the most singular behavior in the temperature dependence of the various susceptibilities, as shown in Fig. 2 for values of $t_{\perp}^{\prime}$ below and above the threshold for superconductivity.

An alternative way to determine the nature of the ordered phase is to look at the wave vector dependence of the renormalized vertex functions close to the divergence. In Fig. 3, we have plotted the SDW vertex function $\Gamma_{S}\left(-k_{\perp}^{\prime}, k_{\perp}^{\prime}, k_{\perp},-k_{\perp}\right)$ in the $k_{\perp}, k_{\perp}^{\prime}$ plane. In the SDW regime, only processes involving particle-hole pairs at wave vector $\left(-k_{\perp}^{\prime}\right)-k_{\perp} \simeq Q_{\perp 0}$ are found to be singular. On the other hand, near the superconducting transition temperature, we obtain the separable form

$$
\Gamma_{S}\left(-k_{\perp}^{\prime}, k_{\perp}^{\prime}, k_{\perp},-k_{\perp}\right) \propto \cos k_{\perp}^{\prime} \cos k_{\perp} .
$$


(a)

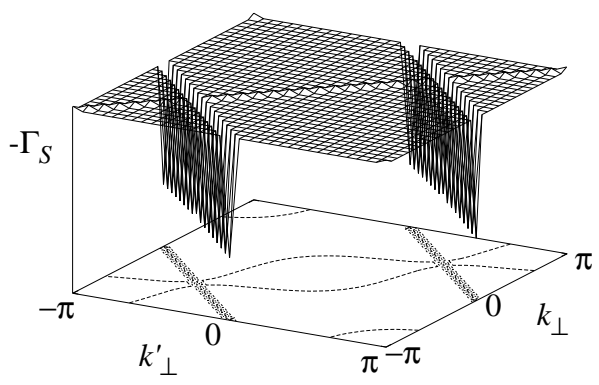

(b)

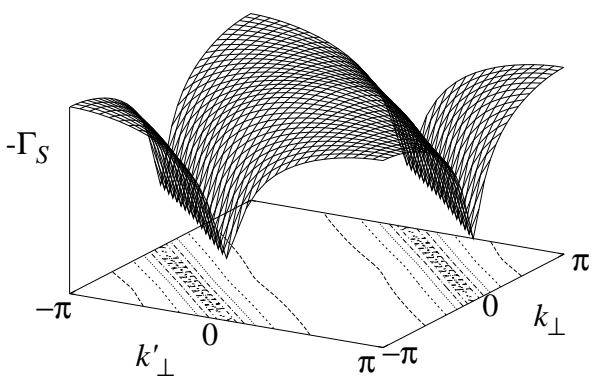

(c)

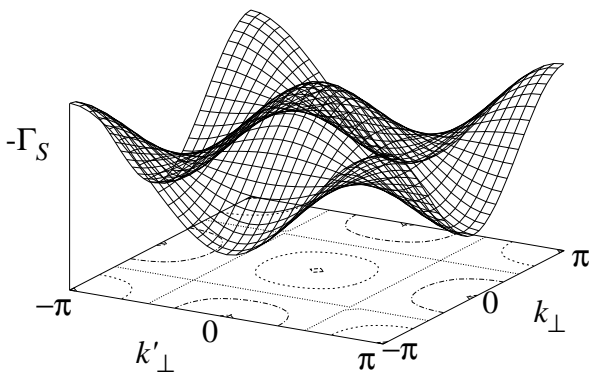

FIG. 3: $-\tilde{\Gamma}_{S}\left(-k_{\perp}^{\prime}, k_{\perp}^{\prime}, k_{\perp},-k_{\perp}\right)$ in the SDW regime $\left(t_{\perp}^{\prime}=\right.$ $\left.0.04 t_{\perp}\right)$ close to the divergence $(\mathrm{a})$, in the $\mathrm{SC} d$ regime $\left(t_{\perp}^{\prime}=\right.$ $\left.0.048 t_{\perp}\right)$ at an intermediate stage of the flow (b) and close to the divergence (c). The vertical scale is arbitrary.

A plot of the singlet interaction amplitude $\Gamma_{s}$ gives essentially the same picture. From Eq. (27), it is clear that the most divergent vertex part $\zeta$ in such a situation is the one proportional to $\cos k_{\perp}$.

Recall that the vertex functions $\Gamma$ we calculate in our $\mathrm{RG}$ scheme are equal to the effective interactions $g^{\text {eff }}$ of a Wilsonian low energy effective theory. Quite early during the flow, the vertex associated to the spin density, $\Gamma_{S}\left(k_{j \perp}\right)$, and hence $g_{S}\left(k_{j \perp}\right)$, develops a peak structure similar to the one close to the SDW transition, but less pronounced, cf. Fig. B(b). There are thus important spin fluctuations at temperature or energy scales above the transition temperature for superconductivity, as is confirmed by the behavior of the associated susceptibility, Fig. 2(b). The same peak structure appears in the effective interaction between electrons forming a singlet (or triplet) pair, $g_{s(t)}\left(k_{j \perp}\right)$. We can decompose the peak of $g_{s}\left(-k_{\perp}^{\prime}, k_{\perp}^{\prime}, k_{\perp},-k_{\perp}\right)$ at intermediate cutoff $\Lambda$ in terms of the variables $\left(k_{\perp}+k_{\perp}^{\prime}\right)$ and $\left(k_{\perp}-k_{\perp}^{\prime}\right)$. Neglecting the weak $\left(k_{\perp}-k_{\perp}^{\prime}\right)$ dependence, the result is, schematically,

$$
\begin{aligned}
& g_{s}\left(-k_{\perp}^{\prime}, k_{\perp}^{\prime}, k_{\perp},-k_{\perp}\right) \\
= & -\frac{1}{N_{\perp}} \sum_{n=-\frac{N_{\perp}}{2}+1}^{\frac{N_{\perp}}{2}} a_{n} e^{i\left(k_{\perp}^{\prime}+k_{\perp}-\pi\right) n} \\
= & -\frac{1}{N_{\perp}} \sum_{n=-\frac{N_{\perp}}{2}+1}^{\frac{N_{\perp}}{2}} a_{n}(-1)^{n} \\
& \times\left(\cos n k_{\perp}^{\prime} \cos n k_{\perp}-\sin n k_{\perp}^{\prime} \sin n k_{\perp}\right) .
\end{aligned}
$$

$|n|$ corresponds to the distance between the chains where the two interacting electrons are located. In the SDW regime, Fig. 31(a), $g_{s}$ has the form of a $\delta$ peak, so that all $a_{n}>0$ will be equal. In the superconducting regime, Fig. 3(b), the peak is slightly enlarged, so that $a_{n}>0$ will be some decreasing function of $|n|$. We thus see that, due to the spin fluctuations, the effective interaction between particles forming a singlet pair contains attractive contributions at all chain distances. (Note that, according to our definitions Eqs. (29) and (30), an "attractive" interaction corresponds to positive $g_{s, t}$.) The most important one is the nearest-neighbor chain one, $\cos k_{\perp}(\mathrm{SC} d)$, followed by $\sin 2 k_{\perp}(\mathrm{SC} g), \cos 3 k_{\perp}$ etc., as it can also be seen from the calculation of the related pairing susceptibilities, cf. Fig. 2(b). Note that, according to the relations (31), $g_{S}$ also gives an attractive contribution to the triplet channel. However, all the three components of a - spin-one boson - SDW fluctuation contribute to the superconducting coupling in the singlet channel, whereas only one contributes to the triplet channel. Antiferromagnetic fluctuations thus favor singlet pairing as compared to triplet pairing. On the other hand, according to Eq. (31), strong charge fluctuations should in principle be able to change this tendency.

\section{INTERCHAIN INTERACTIONS AND TRIPLET SUPERCONDUCTIVITY: INCOMMENSURATE CASE}

In this section, we examine the role of interchain interactions in the phase diagram of the extended quasi-1D electron gas model. We will first consider the influence of interchain backward scattering $g_{1}^{\perp}$ and forward scattering $g_{2}^{\perp}$ separately, before studying their combined effect. The intrachain interactions are kept fixed to their values used in the previous section. Concerning the nesting quality, the overall picture remains the same. For weak deviations from perfect nesting, we find density-wave instabilities, superconductivity for more important deviations, and - discarding possible Kohn-Luttinger effects at low temperatures beyond the numerical accuracy of our RG calculations - a metallic phase when the nesting is deteriorated even more. 


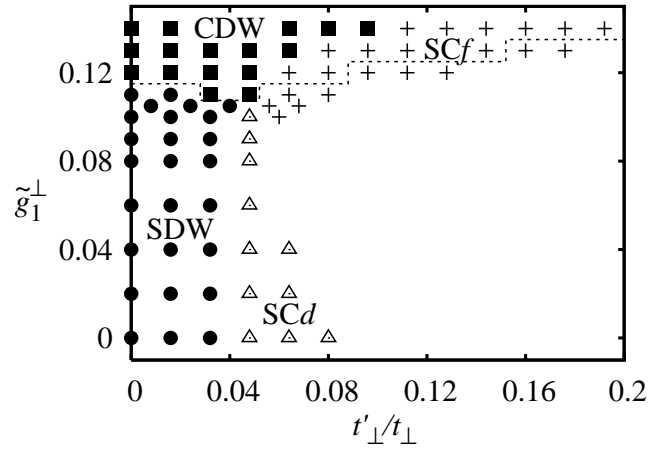

FIG. 4: Low temperature phases versus $g_{1}^{\perp}$, keeping $g_{2}^{\perp}=0$ and $\tilde{g}_{3}=0$. Circles indicate a SDW phase, squares a CDW phase, triangles $\mathrm{SC} d\left(\cos k_{\perp}\right)$ and crosses $\mathrm{SC} f\left(r \cos k_{\perp}\right)$. In the region below the dotted line, spin fluctuations dominate over charge fluctuations in the normal phase.

By switching on $g_{1}^{\perp}$ gradually on the positive scale, one observes that at small values of $g_{1}^{\perp}$, the SDW phase remains essentially unaffected, whereas the transition temperature for $d$-wave superconductivity is considerably re- duced by the presence of a finite $g_{1}^{\perp}$ (Fig. [5), and the region where superconductivity is stable shrinks (Fig. (4). For higher $g_{1}^{\perp}\left(g_{1}^{\perp}\right.$ of the order of $\frac{1}{3}$ of the intrachain backscattering $\left.g_{1}\right), d$-wave superconductivity turns out to be no longer stable and an $f$-wave triplet superconducting phase appears. In this parameter range, $\left(2 k_{F}, \pi\right)$ charge fluctuations are strongly enhanced by interchain backward scattering (Fig. 6). However, spin fluctuations remain important, and in a sizeable region of the phase diagram, triplet superconductivity is preceded in temperature by dominant spin fluctuations in the normal state (Figs. 4 and 6). For the values of intrachain interactions used in this section, and $\tilde{g}_{1}^{\perp}=0.105$, one finds a max$\operatorname{imum} T_{c}^{0}(\mathrm{SC} f) \approx 0.001 t_{\perp} \sim 0.2 \mathrm{~K}$, that is of the same order of magnitude as that of the $d$-wave case. $T_{c}(\mathrm{SC} f)$ increases with the amplitude of $g_{1}^{\perp}$, and the superconducting phase widens (Figs. 4 and 5 . Once triplet superconductivity occurs, $g_{1}^{\perp}$ starts to affect the density wave phase: The SDW state is suppressed and replaced by a CDW. The values of $\tilde{g}_{1}^{\perp}$ for which SC $f$ and CDW phases first appear depend on the values of intrachain interactions and increase with the value of the ratio $g_{1} / g_{2}$.

The origin of the $f$-wave SC and CDW phases can be understood by considering the contribution of the $g_{j}^{\perp}$ 's to the (bare) scattering amplitudes in the singlet and triplet particle-particle channels, as well as in the charge and spin channels:

$$
\begin{gathered}
g_{C}^{\perp}\left(k_{\perp}^{\prime}+q_{\perp}, k_{\perp}-q_{\perp}, k_{\perp}^{\prime}, k_{\perp}\right)=-4 g_{1}^{\perp} \cos q_{\perp}+2 g_{2}^{\perp} \cos \left(q_{\perp}+k_{\perp}^{\prime}-k_{\perp}\right), \\
g_{S}^{\perp}\left(k_{\perp}^{\prime}+q_{\perp}, k_{\perp}-q_{\perp}, k_{\perp}^{\prime}, k_{\perp}\right)=2 g_{2}^{\perp} \cos \left(q_{\perp}+k_{\perp}^{\prime}-k_{\perp}\right), \\
g_{s}^{\perp}\left(-k_{\perp}^{\prime}, k_{\perp}^{\prime}, k_{\perp},-k_{\perp}\right)=2\left(-g_{1}^{\perp}-g_{2}^{\perp}\right) \cos k_{\perp}^{\prime} \cos k_{\perp}+2\left(g_{1}^{\perp}-g_{2}^{\perp}\right) \sin k_{\perp}^{\prime} \sin k_{\perp}, \\
g_{t}^{\perp}\left(-k_{\perp}^{\prime}, k_{\perp}^{\prime}, k_{\perp},-k_{\perp}\right)=2\left(g_{1}^{\perp}-g_{2}^{\perp}\right) \cos k_{\perp}^{\prime} \cos k_{\perp}+2\left(-g_{1}^{\perp}-g_{2}^{\perp}\right) \sin k_{\perp}^{\prime} \sin k_{\perp} .
\end{gathered}
$$

From these equations, it can be easily seen that the interchain repulsion $g_{1}^{\perp}$ contributes positively to $g_{C}^{\perp}$ at $q_{\perp}=\pi$, and therefore induces CDW correlations with a phase difference of $\pi$ between neighboring chains. In the Cooper channel, $g_{1}^{\perp}$ favors triplet $f$-wave and singlet $d_{x y}$-wave pairing, whereas its contribution to singlet $d_{x^{2}-y^{2}}$-wave and triplet $p_{y}$-wave pairing is negative. As for $g_{2}^{\perp}$, it tends to suppress both singlet and triplet pairings on nearest-neighbor chains.

In addition to this 'direct' contribution there is also an indirect effect due to the exchange of density fluctuations. Upon renormalization, $\left(2 k_{F}, \pi\right) \mathrm{CDW}$ correlations are enhanced beyond the level expected from a mean-field treatment of $g_{1}^{\perp}, \underline{43}$ These CDW fluctuations enhance triplet $f$-wave pairing but suppress singlet pairing, whereas the SDW fluctuations are well known to favor singlet pairing (see Eqs. (31)). Eq. (32) shows that the latter reinforce $d_{x^{2}-y^{2}}$ but suppress $d_{x y}$-wave pairing. Regardless of the values of the interaction constants $g_{1}, g_{2}$ and $g_{2}^{\perp}$, the CDW and triplet $f$-wave phases always appear almost simultaneously when $g_{1}^{\perp}$ increases. This suggests that CDW fluctuations (rather than the direct effect of $g_{1}^{\perp}$ in the Cooper channel [Eqs. (34)]) provide the dominant driving force leading to $f$-wave superconductivity.

We now consider the effect of interchain forward scattering $g_{2}^{\perp}$ alone, setting $g_{1}^{\perp}=0$. From Eqs. (34), it can easily be seen that $g_{2}^{\perp}$ contributes negatively to both $g_{s}^{\perp}$ and $g_{t}^{\perp}$ and then favors the suppression of the nearestneighbor-chain Cooper pairing induced by spin fluctuations. Our results (Figs. 7 and 8) show that this is indeed the case. We have seen, however, in section IID that SDW fluctuations can generate a smaller yet present attractive interaction between electrons on next-nearestneighbor chains, which is not affected by $g_{2}^{\perp}$. It follows that when $d$-wave superconductivity is sufficiently weakened by $g_{2}^{\perp}$, it is replaced by $g$-wave singlet pairing 

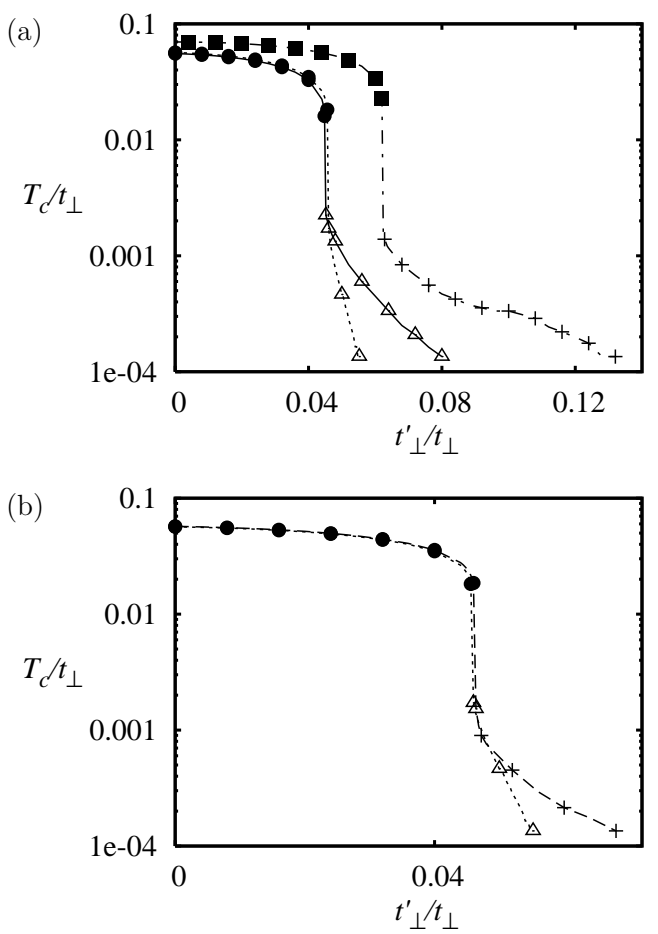

FIG. 5: Transition temperatures for different values of $\tilde{g}_{1}^{\perp}$, when $\tilde{g}_{2}^{\perp}=0$ and $\tilde{g}_{3}=0$ :

(a) $\tilde{g}_{1}^{\perp}=0$ (phase sequence $\mathrm{SDW} \rightarrow \mathrm{SC} d$, continuous line), 0.090 (SDW $\rightarrow \mathrm{SCd}$, dotted line) and $0.120(\mathrm{CDW} \rightarrow \mathrm{SC} f$, dashed-dotted line);

(b) $\tilde{g}_{1}^{\perp}=0.090(\mathrm{SDW} \rightarrow \mathrm{SCd}$, dotted line $)$ and 0.105 (SDW $(\rightarrow \mathrm{SC} d) \rightarrow \mathrm{SC} f$, dashed line). Note that in the latter case, the $\mathrm{SC} d$ phase is extremely narrow.

\section{$\left(\Delta_{r}\left(k_{\perp}\right) \propto r \sin 2 k_{\perp}\right)$. This is shown in Fig. [}

It is worth noting that the instability of the normal state with respect to superconductivity with high angular momentum pairing can be seen as a result of the Kohn-Luttinger effect originally predicted for isotropic metals 37 At variance with more isotropic systems, however, the RG results show that for a quasi-1D metal, the transition temperature of high angular momentum superconducting phases, like $\mathrm{SC} g$, remains experimentally accessible. We find $T_{c, S C g}^{\max } \sim 0.002 t_{\perp} \sim 0.4 \mathrm{~K}$. Finally, the SDW phase remains nearly unaffected by $g_{2}^{\perp}$. This can be understood from Eqs. (33), which indicate that its contribution averages out over the Fermi surface in the particle-hole channel of the RG equations.

We now consider the combined effect of $g_{1}^{\perp}$ and $g_{2}^{\perp}$. Fig. 9] shows the results for $g_{1}^{\perp}=g_{2}^{\perp}$, where all aforementioned phases appear. In the presence of both $g_{1}^{\perp}$ and $g_{2}^{\perp}$, $d$-wave pairing is suppressed even faster than by $g_{1}^{\perp}$ or $g_{2}^{\perp}$ alone. The appearance of $f$-wave pairing is retarded by $g_{2}^{\perp}$ which, as previously mentioned, is detrimental to nearest neighbor chain pairing (Eq. (34)). However, for our choice of intrachain interactions, no triplet phase with pairing on next-nearest-neighbor chains is found for
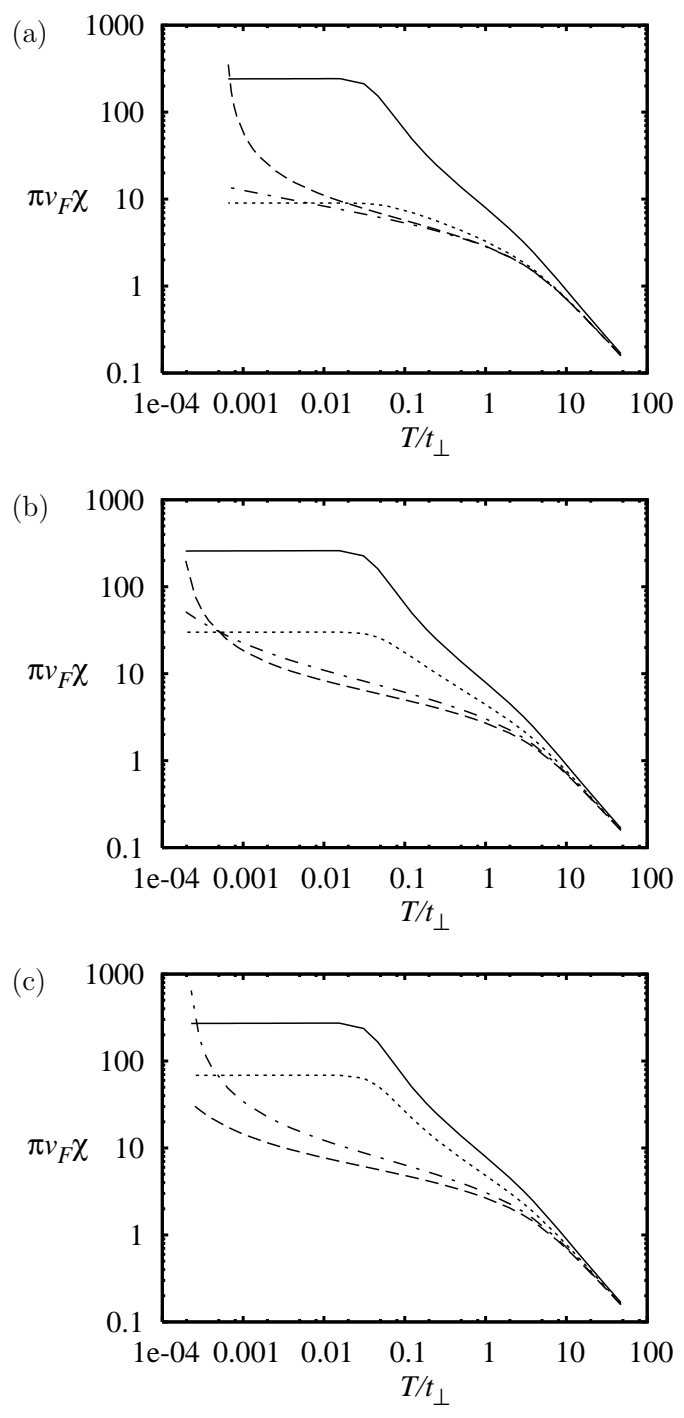

FIG. 6: Temperature dependence of the susceptibilities in the normal phase above the SCd phase ((a) $\tilde{g}_{1}^{\perp}=0$ and (b) $\left.\tilde{g}_{1}^{\perp}=0.08\right)$ and above the SC $f$ phase $\left((\mathrm{c}) \tilde{g}_{1}^{\perp}=0.10\right)$, keeping $\tilde{g}_{2}^{\perp}=0, \tilde{g}_{3}=0$ and $t_{\perp}^{\prime}=0.056 t_{\perp}$. The continuous line corresponds to SDW, the dotted line to CDW, the dashed line to $\mathrm{SC} d$ and the dashed-dotted line to $\mathrm{SC} f$ correlations.

$g_{1}^{\perp}=g_{2}^{\perp}$, since $g_{1}^{\perp}$ favors nearest-neighbor triplet pairing at the outset. As for CDW's, they are found to occur at slightly higher values of $g_{1}^{\perp}$ in the presence of a finite $g_{2}^{\perp}$. As mentioned before, $g_{2}^{\perp}$ has no important effect in the particle-hole channel alone. The slight suppression of the CDW due to $g_{2}^{\perp}$ must therefore come from the 1D regime, where the correlation channels are coupled. This can be checked from the RG equations for $t_{\perp}=0$ given in appendix $\mathrm{A}$

The phase diagram (Fig.9) depends quantitatively and qualitatively on the bare intrachain interactions. When the ratio $g_{1} / g_{2}$ increases, higher values of the interchain interactions are necessary for obtaining CDW and SC $f$ 


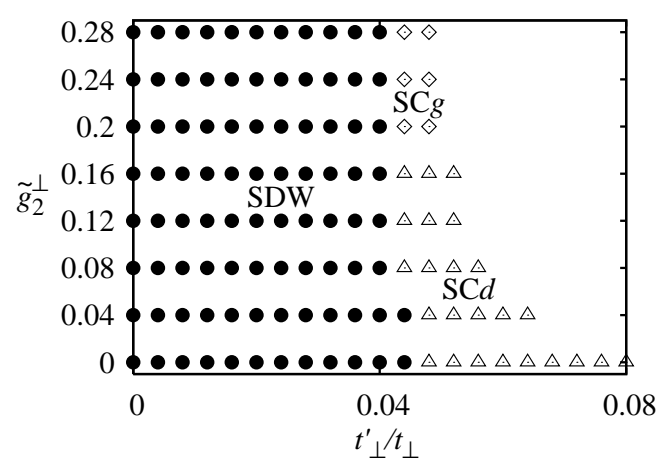

FIG. 7: Low temperature phases for $g_{2}^{\perp}$ varying, keeping $g_{1}^{\perp}=$ 0 and $\tilde{g}_{3}=0$. Circles indicate a SDW phase, triangles SCd and diamonds $\mathrm{SC} g\left(r \sin 2 k_{\perp}\right)$.

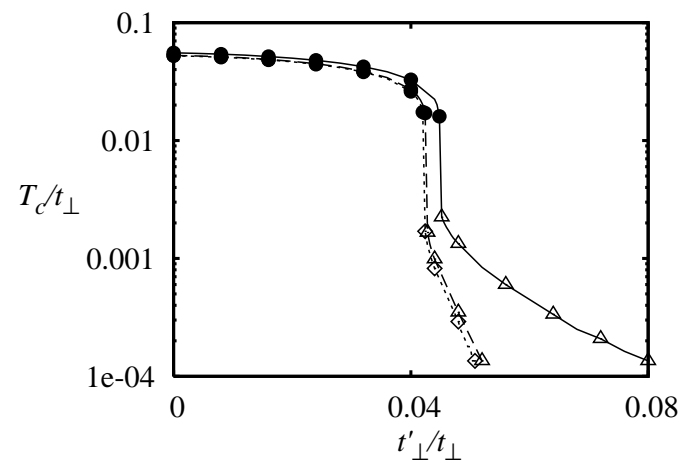

FIG. 8: Transition temperatures for different values of $\tilde{g}_{2}^{\perp}$, if $\tilde{g}_{1}^{\perp}=0$ and $\tilde{g}_{3}=0: \quad \tilde{g}_{2}^{\perp}=0$ (SDW $\rightarrow \mathrm{SC} d$, continuous line), 0.16 (SDW $\rightarrow \mathrm{SC} d$, dashed line) and 0.20 (SDW $\rightarrow \mathrm{SC} g$, dotted line).

phases because $\Gamma_{C}$ is more negative at the outset. For $g_{1}=g_{2}$, we even find a triplet SC $f$ order parameter of the form $\Delta_{r}\left(k_{\perp}\right) \propto \sin 2 k_{\perp}$, corresponding to second-nearestneighbor chain pairing, instead of $\Delta_{r}\left(k_{\perp}\right) \propto r \cos k_{\perp}$. This is coherent with the fact that, in the $1 \mathrm{D}$ regime, the renormalization of $\Gamma_{1}^{\perp}$ is proportional to $\Gamma_{1}^{\perp} \Gamma_{C}$, so that a more negative $\Gamma_{C}$ reduces $\Gamma_{1}^{\perp}$ and hence nearest-neighbor chain triplet pairing (appendix A).

\section{EFFECT OF UMKLAPP PROCESSES}

Conductors like the Bechgaard and Fabre salts are slightly dimerized in the direction of the organic chains. It follows that at low energy or temperature, the hole band can be considered as effectively half filled rather than quarter-filled and this gives rise to Umklapp scattering processes with amplitudes $g_{3}$ and $g_{3}^{\perp}$. To leading order, the bare amplitude of $g_{3} \approx g_{1} \Delta_{D} / E_{F}$ is proportional to the dimerization gap $\Delta_{D}, 4.7 .67$ which yields a $g_{3}$ that is rather weak as compared to $g_{1,2}$. We assume a similar ratio between the interchain couplings $g_{3}^{\perp}$ and

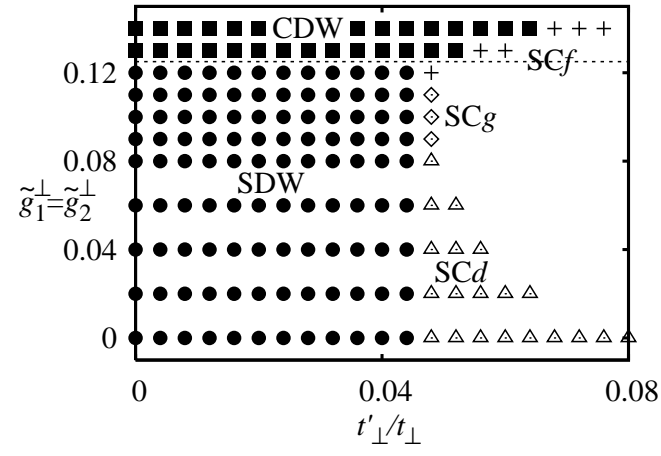

FIG. 9: Low temperature phases for $g_{1}^{\perp}=g_{2}^{\perp}, g_{3}=0$. Circles indicate a SDW phase, black squares a CDW phase, triangles $\mathrm{SC} d\left(\cos k_{\perp}\right)$, white diamonds $\mathrm{SC} g\left(r \sin 2 k_{\perp}\right)$ and crosses $\mathrm{SC} f\left(r \cos k_{\perp}\right)$. Below the dotted line, spin fluctuations dominate over charge fluctuations in the normal phase.

$g_{1,2}^{\perp} 68$

Intrachain Umklapp processes enhance the formation of 'site' centered SDW correlations and 'bond' centered CDW correlations and weaken bond SDW and site CDW fluctuations, as can be seen from Eqs. (25). Thus, contrary to the incommensurate case, site and bond densitywave orders no longer join together and must be considered separately with their distinct strengths at half filling. In addition, intrachain Umklapp scattering reinforces the effective interactions $g_{C}$ and $g_{S}$ at momentum transfers $\left(2 k_{F}, \pi\right)$, thus increasing spin and charge density fluctuations of both site and bond type. These effects are known in the 1D case (see e. g. Ref. 69 and also Appendix A, as well as from calculations restricted to the particle-hole channels (Ref. 70 and Appendix A). Even though Umklapp processes do not enter the particle-particle channel directly, they do enhance Cooper pairing, because they amplify the peak at $\left(2 k_{F}, \pi\right)$ in the effective interactions $g_{S, C}$ and thus the attraction between electrons on neighboring chains [see Eq. (32)]. Since density-wave correlations are enhanced with respect to the incommensurate case, transition temperatures are also higher ${ }^{20}$ This is true for the formation of density-wave states as well as for superconductivity (Fig. 10(a)). The ratio $T_{c}^{0} / T_{c, S C}^{\max }$ remains essentially unaffected by $g_{3}$. The critical $t_{\perp c}^{\prime}$ needed to destroy the density-wave phase is also higher in the presence of Umklapp scattering.

Let us now turn to the effect of interchain interactions in the presence of Umklapp processes. We will first consider the effect of a finite intrachain $g_{3}$, for $g_{1}^{\perp}=g_{2}^{\perp}>0$ and $g_{3}^{\perp}=0$. A comparison of Figs. 9] and [11(a) shows that the regions of $d$-wave and $f$-wave superconductivity are now enlarged, and there is no more $\mathrm{SC} g$ phase corresponding to next-nearest-neighbor chain singlet pairing. This is a consequence of the fact that density-wave correlations are reinforced by Umklapp scattering. A stronger $g_{2}^{\perp}$ would then be needed to destroy nearest-neighbor chain pairing, but owing to the condition $g_{1}^{\perp}=g_{2}^{\perp}$ the 

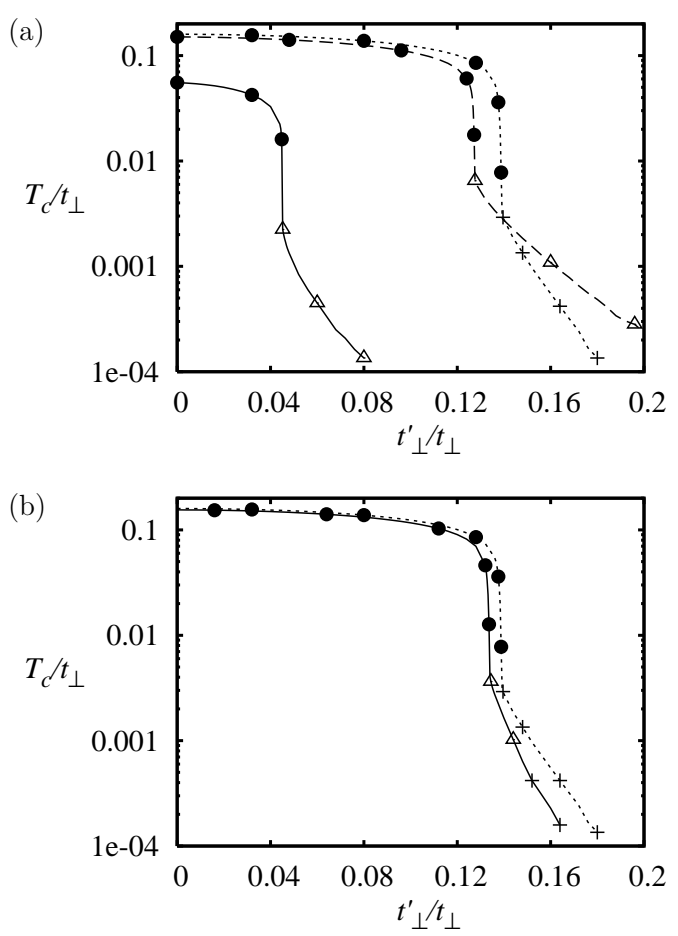

FIG. 10: Transition temperatures when Umklapp processes are taken into account.

(a) Without interchain Umklapp scattering: $\tilde{g}_{3}=0, \tilde{g}_{j}^{\perp}=0$ (SDW $\rightarrow \mathrm{SC} d$, continuous line),

$\tilde{g}_{3}=0.02, \tilde{g}_{j}^{\perp}=0(\mathrm{SDW} \rightarrow \mathrm{SC} d$, dashed line $)$,

$\tilde{g}_{3}=0.02, \tilde{g}_{1}^{\perp}=\tilde{g}_{2}^{\perp}=0.1, \tilde{g}_{3}^{\perp}=0,(\mathrm{SDW} \rightarrow \mathrm{SC} f$, dotted line).

(b) Effect of interchain Umklapp processes: $\tilde{g}_{3}=0.02, \tilde{g}_{1}^{\perp}=$ $\tilde{g}_{2}^{\perp}=0.1, \tilde{g}_{3}^{\perp}=0$ (SDW $\rightarrow \mathrm{SC} f$, dotted line) and

$\tilde{g}_{3}^{\perp}=\frac{\tilde{g}_{1}}{\tilde{g}_{3}} \tilde{g}_{1}^{\perp}(\mathrm{SDW} \rightarrow \mathrm{SC} d \rightarrow \mathrm{SC} f$, continuous line $)$.

$\mathrm{SC} f$ phase is also reinforced and in turn stabilized once the $\mathrm{SC} d$ phase is suppressed.

When we finally add interchain Umklapp scattering $g_{3}^{\perp}$, the picture does not change significantly (Fig. 11(b)). The only effect is that the occurrence of SC $f$ and CDW phases takes place at slightly higher values of the interchain interactions in the phase diagram, whereas transition temperatures are scarcely lower (Fig. 10(b)). In the $1 \mathrm{D}$ regime $\left(\Lambda \gg t_{\perp}\right), g_{3}^{\perp}$ enhances $g_{C}$ (appendix $\mathrm{A}$ ), but this is only a second order effect. On the other hand, the bare contribution of $g_{3}^{\perp}$, Eq. (6), reduces the peak in the effective interaction $g_{3}\left(k_{1 \perp}^{\prime}, k_{2 \perp}^{\prime}, k_{2 \perp}, k_{1 \perp}\right)$ at $k_{1 \perp}^{\prime}-k_{1 \perp}=\pi$, which is connected to the charge density fluctuations (appendix A). This influence is linear in $g_{3}^{\perp}$ and therefore stronger than the 1D effect, thus explaining the overall weakening of CDW and, in turn, of SC $f$ correlations. There is no direct effect of $g_{3}^{\perp}$ on SDW correlations. However, the RG equations (Appendix A) show that $g_{3}^{\perp}$ weakens the renormalization of $g_{3}$ in the $1 \mathrm{D}$ regime and in turn the increase of transition temperatures in comparison to the incommensurate situation.

In Fig. 12 we show the typical behavior of the most

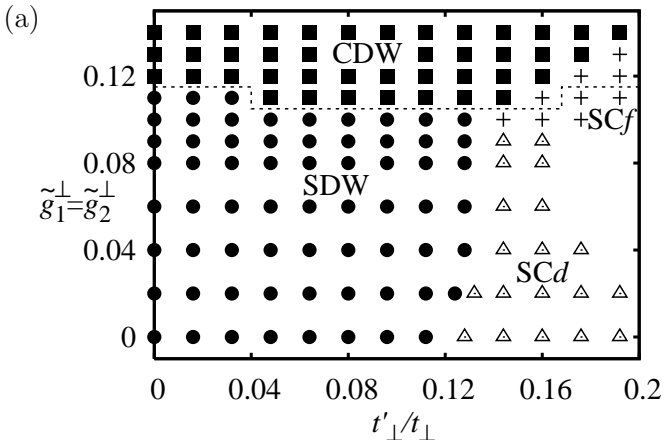

(b)

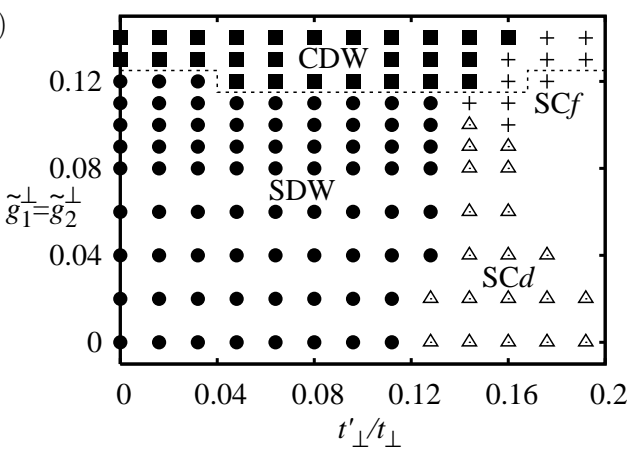

FIG. 11: Low temperature phases in the presence of Umklapp processes, with $g_{3}^{\perp}=0$ (a) and $g_{3}^{\perp} / g_{3}=g_{1}^{\perp} / g_{1}$ (b), taking $\tilde{g}_{2}^{\perp}=\tilde{g}_{1}^{\perp}$. Circles indicate a site-SDW phase, squares a bond-CDW phase, triangles $\mathrm{SC} d\left(\cos k_{\perp}\right)$ and crosses $\mathrm{SC} f$ $\left(r \cos k_{\perp}\right)$. Below the dotted lines, spin fluctuations dominate over charge fluctuations in the normal phase.

important susceptibilities as a function of temperature in the normal phase above the superconducting phases. Fig. 12(a) shows that for sufficiently important interchain interactions, triplet correlations are already strongly enhanced, although the ordered phase still corresponds to spin singlet pairing. SDW correlations are always important. They are the dominant fluctuations in the normal phase in the major part of the parameter range we have explored, sometimes even above the triplet SC phase (cf. Figs. 12(c) and [11).

\section{DISCUSSION AND CONCLUSION}

In this work, we have determined the possible electronic phases of the extended quasi-1D electron gas model that includes both intrachain and interchain repulsive interactions, interchain electron hopping and the influence of nesting deviations. Our results reveal that in correlated quasi-1D metals both for zero and non zero Umklapp scattering, interchain interactions can act as a key factor in expanding the range of possibilities of ordered states compared to the case where only repulsive intrachain interactions are present $19,20,21$ At large momentum transfer, the interchain electron-electron cou- 

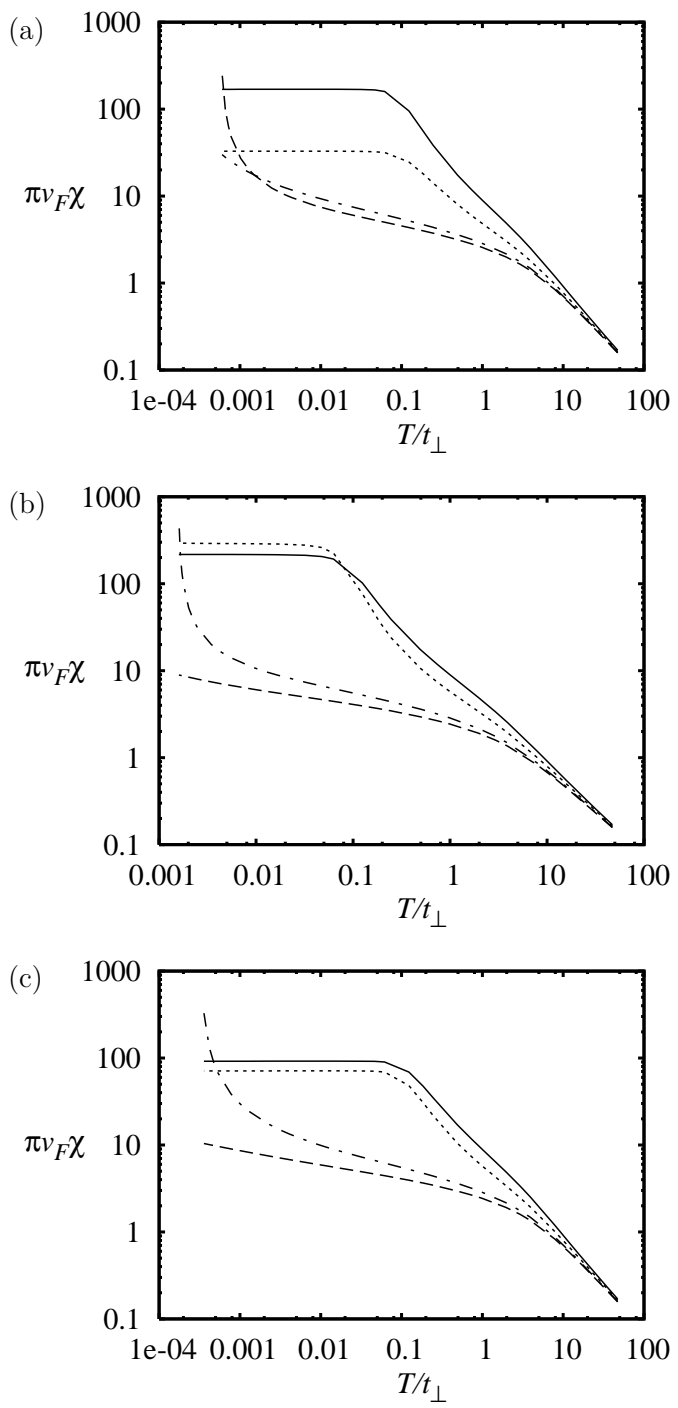

FIG. 12: Temperature dependence of the susceptibilities in the normal phase above the SCd phase ((a) $t_{\perp}^{\prime}=0.152 t_{\perp}$, $\left.\tilde{g}_{1}^{\perp}=0.08\right)$ and above the SC $f$ phase $\left((\mathrm{b}) t_{\perp}^{\prime}=0.152 t_{\perp}\right.$ and (c) $t_{\perp}^{\prime}=0.176 t_{\perp}$, both for $\tilde{g}_{1}^{\perp}=0.12$ ), taking $\tilde{g}_{3}=0.02$ and $\tilde{g}_{2}^{\perp}=\tilde{g}_{1}^{\perp}=\frac{\tilde{g}_{1}}{\tilde{g}_{3}} \tilde{g}_{3}^{\perp}$. The continuous line corresponds to SDW, the dotted line to CDW, the dashed line to SCd and the dashed-dotted line to SC $f$ correlations.

pling acts as a short-range interaction that is responsible for the enhancement of CDW correlations, consistently with what was found long ago in the absence of interchain hopping. ${ }^{41.44}$ For finite $t_{\perp}$, however, interchain interaction leaves the amplitude of SDW correlations essentially unaffected and a relatively small critical value of $g_{1}^{\perp}$ coupling is then needed to make the CDW ordered state possible, and this, on equal footing with the SDW phase, which is known to dominate the phase diagram when only repulsive intrachain interactions are present. When nesting deviations are cranked up beyond some threshold value $t_{\perp c}^{\prime}$, density-wave order is suppressed and interchain interactions turn out to affect correlations of the Cooper channel too. Thus an important conclusion that emerges from this work is the gradual suppression of interchain $d$-wave pairing when repulsive shortrange interchain interaction increases. As a result of the growth of CDW correlations in the normal state, it ultimately yields the stabilization of a triplet SC $f$ superconducting phase corresponding to an order parameter $\Delta_{r}\left(k_{\perp}\right)=r \Delta \cos k_{\perp}$ having nodes on the warped Fermi surface that are at the same locus as for the SCd case. The normal phase is still dominated by strong SDW fluctuations over a sizable region of the phase diagram in this sector.

It is interesting to consider how far the RG results of this work are applicable to quasi-1D organic conductors. In the case of the Bechgaard (TMTSF) ${ }_{2} \mathrm{X}$ salts with centrosymmetric anions $\mathrm{X}$ for example, the observation of a SDW-CDW coexistence below the critical pressure for superconductivity ${ }^{48,50,71}$ indicates that interchain electron repulsion is a relevant interaction in these materials besides intrachain interactions and interchain hopping. Accordingly, the RG calculations show that a relatively small and realistic amplitude of repulsive interchain interaction is sufficient to bring the stability of CDW order close to SDW, indicating that this part of interaction would indeed play an important role in the emergence of density-wave order in these materials. The suppression of the SDW state (and presumably of CDW as well) followed by the emergence of superconductivity is well known to constitute the closing sequence of transitions that characterizes virtually all members of the Bechgaard and Fabre salts series as one moves along the pressure scale. Since pressure introduces alterations of the electron spectrum, it prompts deviations from perfect nesting. In our model, these simulate the main effect of pressure, which together with a reasonable set of parameters, yield a 'pressure' profile of the critical temperature that agrees quite well with the characteristic variation seen in experiments ${ }^{72}$ As to the nature of the superconductivity in these materials, our results based on a purely electronic model, indicate that given the observation of the close proximity between SDW and CDW ordered states, not only SCd but also triplet SC $f$ order parameter become serious candidates for the description of the superconducting phase in these compounds (Figs. 49] and 11). However, as pressure also affects the normalized amplitudes $\tilde{g}_{i}^{(\perp)}$ through the band width and the dimerization gap (Umklapp), and $g_{i}^{\perp}$ through the interchain distance,, 4.45 the actual trajectory in the phase diagram under pressure cannot be determined with great precision. It follows that besides the possibilities $\mathrm{SDW} \rightarrow \mathrm{SCd}$ or $\mathrm{SDW} \rightarrow \mathrm{SC} f$, sequence of transitions such as $\mathrm{SDW} \rightarrow$ $\mathrm{SC} d \rightarrow \mathrm{SC} f$, where one can pass from singlet to triplet $\mathrm{SC}$ order under pressure, cannot be excluded. It is worth remarking that in this sector of the phase diagram, the addition of a small magnetic field - as actually used in many experiments 31,32 - or accounting for the small but yet finite spin anisotropy would tend to tip the balance 
in favor of a triplet order parameter ${ }^{21.73}$

Experimental features of the normal phase also argue in favor of this region of the phase diagram for the Bechgaard salts. This is the case of the puzzling growth of CDW correlations seen in optical conductivity in the low temperature part of the metallic phase above the superconducting transition ${ }^{74} \mathrm{CDW}$ correlations are found to be significantly enhanced in a temperature region where NMR experiments reveal the existence of strong SDW correlations. ${ }^{13,14}$ This feature cannot be captured for realistic intrachain interactions alone. It can find, however, a natural explanation in the framework of the extended quasi-1D electron gas model, for which interchain interactions can boost the amplitude of CDW correlations besides those of the SDW channel that are kept essentially unchanged.

In the case of non-centrosymmetric anions (e. g. $\mathrm{X}=\mathrm{ClO}_{4}$ ), our approach should be refined in order to take into account the doubling of the unit cell in the transverse direction due to the anion ordering taking place below $24 \mathrm{~K}$ in the normal phase. The concomittant reduction of the Brillouin zone yields two electronic bands at the Fermi level and in turn multiple nesting vectors. ${ }^{75.76}$ An accurate description of this Fermi surface should be incorporated in the RG approach, and a modification of the node structure for the SC gap is expected. ${ }^{77} \mathrm{~A}$ previous (simplified) RPA-like calculation has shown that the nodes of a $d$-wave SC order parameter, which are located at $k_{\perp}= \pm \pi / 2$, are precisely found where a gap opens due to $\mathrm{ClO}_{4}$ anion ordering, thus making the $\mathrm{SC}$ phase effectively nodeless at low temperature 73

The results presented in this work may also be relevant for the phase diagram of other series of quasi-1D organic superconductors. In this matter, the case of the twochain compounds TTF $\left[\mathrm{M}(\text { dmit })_{2}\right]_{2}(\mathrm{M}=\mathrm{Ni}, \mathrm{Pd})$ is of particular interest. These compounds are characterized by an incommensurate CDW state that takes place on the $\mathrm{M}(\mathrm{dmit})_{2}$ stacks at low pressure ${ }^{78}$ At some critical pressure, they become superconducting. ${ }^{79,80}$ The variation of the critical temperature under pressure has been analyzed in detail in the case of $\mathrm{M}=\mathrm{Pd}$, which shows close similarity with the one found for the Bechgaard and Fabre salts ${ }^{80}$ The temperature scale for the CDW instability at low pressure is relatively large, however, and owing to the pronounced anisotropy of the band parameters in these compounds, $\stackrel{78,81}{~ t h i s ~ i n d i c a t e s ~ t h a t ~ t h e ~}$ interchain interaction is likely to be a key coupling in the stabilization of a 3D ordered state in these materials. According to our model, these conditions would be favorable to the existence of a triplet SC $f$ state in these systems under pressure (see e.g. Fig. 9).

Another result that is highlighted by our analysis in the incommensurate case is the occurrence of a singlet $\mathrm{SC} g$ state when the interchain electron scattering dominates for small momentum transfer. The $g_{2}^{\perp}$ coupling tends to suppress electron pairing between the first nearestneighbor chains, which therefore suppresses SCd or SC $f$ type of superconductivity. However, an instability of the normal state remains possible. It results from the oscil- lating tail of density-wave correlations in the transverse direction which favors longer range pairing between electrons separated by more than one interchain distance. The fact that an instability of the normal state persists in the Cooper channel illustrates how the quasi-1D geometry for electrons, with its inherent interference between Peierls and Cooper channels, is prone to magnify the Kohn-Luttinger mechanism for the (Cooper) instability of a Fermi liquid for repulsive interactions $\stackrel{37}{37}$ One can easily infer from our results that the addition of longer range interchain interactions will frustrate short-range interchain pairing and shift it to larger interchain distances, thus unfolding possibilities of superconductivity at even larger angular momentum pairing.

\section{Acknowledgments}

J. C. N. is grateful to the Gottlieb Daimler- und Karl Benz-Stiftung for partial support. C. B. thanks D. Jérome, Y. Fuseya, M. Tsuchiizu, Y. Suzumura, L. G. Caron and S. Brown for useful discussions and comments, and the Natural Sciences and Engineering Research Council of Canada (NSERC), le Fonds Québecois de la Recherche sur la Nature et les Technologies du Gouvernement du Québec (FQRNT), and the Institut Canadien de Recherches Avancées (CIAR) for financial support. N. D. thanks the condensed matter theory group of the Université de Sherbrooke for its hospitality.

\section{APPENDIX A: RG EQUATIONS IN LIMITING CASES}

In order to obtain the one-dimensional limit of the RG equations given in section $1 \mathrm{~B}$, we neglect interchain hopping $\left(t_{\perp}=t_{\perp}^{\prime}=0\right)$ and take into account intrachain and nearest-neighbor-chain interactions only. We thus obtain 41,44

$$
\begin{aligned}
\dot{\tilde{\Gamma}}_{1}^{(1 D)} & =-\left(\tilde{\Gamma}_{1}^{(1 D)}\right)^{2}-2\left[\left(\tilde{\Gamma}_{1}^{\perp}\right)^{2}+\left(\tilde{\Gamma}_{3}^{\perp}\right)^{2}\right] \\
\dot{\tilde{\Gamma}}_{1}^{\perp} & =\tilde{\Gamma}_{1}^{\perp}\left[-2 \tilde{\Gamma}_{1}^{(1 D)}+\tilde{\Gamma}_{2}^{(1 D)}-\tilde{\Gamma}_{2}^{\perp}\right]-\tilde{\Gamma}_{3}^{(1 D)} \tilde{\Gamma}_{3}^{\perp} \\
\dot{\tilde{\Gamma}}_{2}^{(1 D)} & =-\frac{1}{2}\left(\tilde{\Gamma}_{1}^{(1 D)}\right)^{2}+\frac{1}{2}\left(\tilde{\Gamma}_{3}^{(1 D)}\right)^{2} \\
\dot{\tilde{\Gamma}}_{2}^{\perp} & =-\frac{1}{2}\left(\tilde{\Gamma}_{1}^{\perp}\right)^{2}+\frac{1}{2}\left(\tilde{\Gamma}_{3}^{\perp}\right)^{2} \\
\dot{\tilde{\Gamma}}_{3}^{(1 D)} & =\tilde{\Gamma}_{3}^{(1 D)}\left[-\tilde{\Gamma}_{1}^{(1 D)}+2 \tilde{\Gamma}_{2}^{(1 D)}\right]-4 \tilde{\Gamma}_{1}^{\perp} \tilde{\Gamma}_{3}^{\perp} \\
\dot{\Gamma}_{3}^{\perp} & =\tilde{\Gamma}_{3}^{\perp}\left[-2 \tilde{\Gamma}_{1}^{(1 D)}+\tilde{\Gamma}_{2}^{(1 D)}+\tilde{\Gamma}_{2}^{\perp}\right]-\tilde{\Gamma}_{3}^{(1 D)} \tilde{\Gamma}_{1}^{\perp} .
\end{aligned}
$$

Alternatively, we may restrict the renormalization to a given correlation channel in certain situations. For the particle-hole channel, it is convenient to rewrite the RG equations in terms of the vertex functions $\Gamma_{C, S}$ and $\Gamma_{3}^{(C, S)}$, as defined in eqs. 29). Remember however that $\Gamma_{3}^{(C)}$ and $\Gamma_{3}^{(S)}$ are not independent: 
(a)

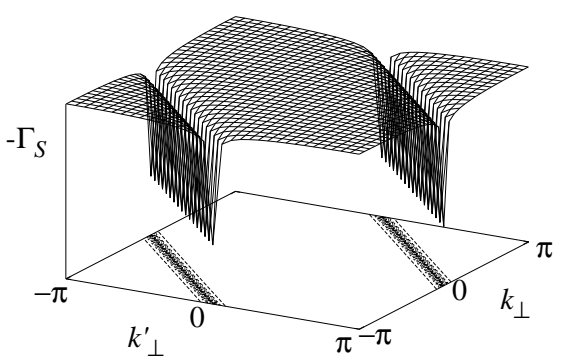

(b)

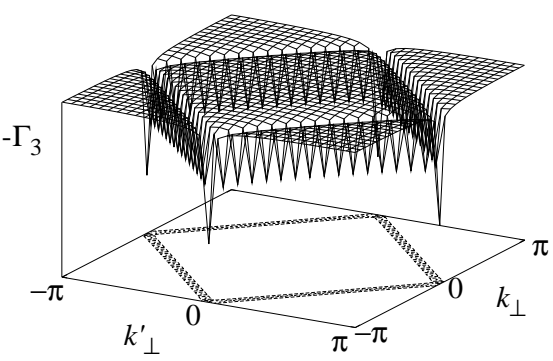

FIG. 13: Typical pictures of the interactions $\Gamma_{S}\left(-k_{\perp}^{\prime}, k_{\perp}^{\prime}, k_{\perp},-k_{\perp}\right)$ (a) and $\Gamma_{3}\left(-k_{\perp}^{\prime}, k_{\perp}^{\prime}, k_{\perp},-k_{\perp}\right)$ (b), for initial values $\tilde{g}_{S}=\tilde{g}_{C}=0.2, \tilde{g}_{3}=0.02$, if the renormalization is restricted to the Peierls channel. The result for $\Gamma_{C}$ is identical to that for $\Gamma_{S}$.

$$
\Gamma_{3}^{(C)}\left(k_{1 \perp}^{\prime}, k_{2 \perp}^{\prime}, k_{2 \perp}, k_{1 \perp}\right)=-2 \Gamma_{3}^{(S)}\left(k_{1 \perp}^{\prime}, k_{2 \perp}^{\prime}, k_{1 \perp}, k_{2 \perp}\right)+\Gamma_{3}^{(S)}\left(k_{1 \perp}^{\prime}, k_{2 \perp}^{\prime}, k_{2 \perp}, k_{1 \perp}\right) .
$$

We obtain

$$
\begin{aligned}
\left.\dot{\tilde{\Gamma}}_{C}\left(k_{1 \perp}^{\prime}, k_{2 \perp}^{\prime}, k_{2 \perp}, k_{1 \perp}\right)\right|_{\mathcal{P}}= & \frac{1}{N_{\perp}} \sum_{k_{\perp}} B_{\mathcal{P}}\left(k_{\perp}, k_{1 \perp}^{\prime}-k_{2 \perp}\right) \\
& \times\left\{\tilde{\Gamma}_{C}\left(k_{1 \perp}^{\prime}, k_{\perp}, k_{2 \perp}, k_{\perp}^{\prime}\right) \tilde{\Gamma}_{C}\left(k_{\perp}^{\prime}, k_{2 \perp}^{\prime}, k_{\perp}, k_{1 \perp}\right)\right. \\
& \left.+\tilde{\Gamma}_{3}^{(C)}\left(k_{1 \perp}^{\prime}, k_{\perp}, k_{2 \perp}, k_{\perp}^{\prime}\right) \tilde{\Gamma}_{3}^{(C)}\left(k_{\perp}^{\prime}, k_{2 \perp}^{\prime}, k_{\perp}, k_{1 \perp}\right)\right\} \\
\left.\dot{\tilde{\Gamma}}_{S}\left(k_{1 \perp}^{\prime}, k_{2 \perp}^{\prime}, k_{2 \perp}, k_{1 \perp}\right)\right|_{\mathcal{P}}= & \frac{1}{N_{\perp}} \sum_{k_{\perp}} B_{\mathcal{P}}\left(k_{\perp}, k_{1 \perp}^{\prime}-k_{2 \perp}\right) \\
& \times\left\{\tilde{\Gamma}_{S}\left(k_{1 \perp}^{\prime}, k_{\perp}, k_{2 \perp}, k_{\perp}^{\prime}\right) \tilde{\Gamma}_{S}\left(k_{\perp}^{\prime}, k_{2 \perp}^{\prime}, k_{\perp}, k_{1 \perp}\right)\right. \\
& \left.+\tilde{\Gamma}_{3}^{(S)}\left(k_{1 \perp}^{\prime}, k_{\perp}, k_{2 \perp}, k_{\perp}^{\prime}\right) \tilde{\Gamma}_{3}^{(S)}\left(k_{\perp}^{\prime}, k_{2 \perp}^{\prime}, k_{\perp}, k_{1 \perp}\right)\right\}
\end{aligned}
$$




$$
\begin{aligned}
\left.\dot{\tilde{\Gamma}}_{3}^{(S)}\left(k_{1 \perp}^{\prime}, k_{2 \perp}^{\prime}, k_{2 \perp}, k_{1 \perp}\right)\right|_{\mathcal{P}}= & \frac{1}{N_{\perp}} \sum_{k_{\perp}} B_{\mathcal{P}}\left(k_{\perp}, k_{1 \perp}^{\prime}-k_{1 \perp}\right) \\
& \times \frac{1}{2} \times\left\{-\tilde{\Gamma}_{C}\left(k_{1 \perp}^{\prime}, k_{\perp}, k_{1 \perp}, k_{\perp}^{\prime}\right) \tilde{\Gamma}_{3}^{(C)}\left(k_{\perp}^{\prime}, k_{2 \perp}^{\prime}, k_{\perp}, k_{2 \perp}\right)\right. \\
& -\tilde{\Gamma}_{3}^{(C)}\left(k_{1 \perp}^{\prime}, k_{\perp}, k_{1 \perp}, k_{\perp}^{\prime}\right) \tilde{\Gamma}_{C}\left(k_{\perp}^{\prime}, k_{2 \perp}^{\prime}, k_{\perp}, k_{2 \perp}\right) \\
& +\tilde{\Gamma}_{S}\left(k_{1 \perp}^{\prime}, k_{\perp}, k_{1 \perp}, k_{\perp}^{\prime}\right) \tilde{\Gamma}_{3}^{(S)}\left(k_{\perp}^{\prime}, k_{2 \perp}^{\prime}, k_{\perp}, k_{2 \perp}\right) \\
& \left.+\tilde{\Gamma}_{3}^{(S)}\left(k_{1 \perp}^{\prime}, k_{\perp}, k_{1 \perp}, k_{\perp}^{\prime}\right) \tilde{\Gamma}_{S}\left(k_{\perp}^{\prime}, k_{2 \perp}^{\prime}, k_{\perp}, k_{2 \perp}\right)\right\} \\
& +\frac{1}{N_{\perp}} \sum_{k_{\perp}} B_{\mathcal{P}}\left(k_{\perp}, k_{1 \perp}^{\prime}-k_{2 \perp}\right) \\
& \times\left\{\tilde{\Gamma}_{S}\left(k_{1 \perp}^{\prime}, k_{\perp}, k_{2 \perp}, k_{\perp}^{\prime}\right) \tilde{\Gamma}_{3}^{(S)}\left(k_{\perp}^{\prime}, k_{2 \perp}^{\prime}, k_{\perp}, k_{1 \perp}\right)\right. \\
& \left.+\tilde{\Gamma}_{3}^{(S)}\left(k_{1 \perp}^{\prime}, k_{\perp}, k_{2 \perp}, k_{\perp}^{\prime}\right) \tilde{\Gamma}_{S}\left(k_{\perp}^{\prime}, k_{2 \perp}^{\prime}, k_{\perp}, k_{1 \perp}\right)\right\} .
\end{aligned}
$$

These equations are remarquably symmetric with respect to spin and charge density correlations, cf. e.g. the results when we take $g_{S}=g_{C}$ from the beginning (Fig. 13). For low temperature and energy cutoff $\Lambda$, the particle-hole loop integral $B_{\mathcal{P}}\left(q_{\perp}\right)$ is strongly peaked for momentum transfers equal to the best nesting vector, $i$. e. $q_{\perp}=\pi$. As can be seen from the preceding equations, this generates a peak at $k_{1 \perp}^{\prime}-k_{2 \perp}=\pi$ in $\Gamma_{S}$ (responsible for the SDW) and in $\Gamma_{C}$ (responsible for the CDW). In $\Gamma_{3}$, peaks are created at $k_{1 \perp}^{\prime}-k_{2 \perp}=\pi$ as well as $k_{1 \perp}^{\prime}-k_{1 \perp}=\pi$. The peak in $\Gamma_{3}$ for $k_{1 \perp}^{\prime}-k_{2 \perp}=\pi$ enhances the spin density correlations, whereas the one for $k_{1 \perp}^{\prime}-k_{1 \perp}=\pi$ supports the charge density correlations.

Let us now consider the influence of interchain interactions [Eq. [6] ] on these structures. Interchain Umklapp scattering $g_{3}^{\perp}$ reduces the latter peak and thus weakens the charge fluctuations, whereas it has no direct influence on the spin correlations. $g_{1}^{\perp}$ and $g_{2}^{\perp}$ have no direct effect on the spin fluctuations either. Interchain backward scattering $g_{1}^{\perp}$, on the contrary, reinforces the peak in $g_{C}$ at $k_{1 \perp}^{\prime}-k_{2 \perp}=\pi$ and thus supports the formation of charge density waves.

We finally give the RG equations restricted to the particle-particle channel. They are most conveniently written in terms of the singlet and triplet pair interactions, $\alpha=s, t$ :

$$
\begin{aligned}
\left.\dot{\tilde{\Gamma}}_{\alpha}\left(k_{\perp 1}^{\prime} k_{\perp 2}^{\prime} k_{\perp 2} k_{\perp 1}\right)\right|_{\mathcal{C}}= & \frac{1}{N_{\perp}} \sum_{k_{\perp}} B_{\mathcal{C}}\left(k_{\perp}, q_{\mathcal{C} \perp}\right) \\
& \times\left.\tilde{\Gamma}_{\alpha}\left(k_{\perp 1}^{\prime} k_{\perp 2}^{\prime} k_{\perp} k_{\perp}^{\prime}\right) \tilde{\Gamma}_{\alpha}\left(k_{\perp} k_{\perp}^{\prime} k_{\perp 1} k_{\perp 2}\right)\right|_{\substack{k_{\perp}^{\prime}=-k_{\perp}+q \mathcal{C} \\
q_{\mathcal{C} \perp}=k_{\perp 1}+k_{\perp 2}}} .
\end{aligned}
$$

1 C. Bourbonnais and D. Jérome, in Advances in Synthetic Metals, Twenty Years of Progress in Science and Technology, edited by P. Bernier, S. Lefrant, and G. Bidan (Elsevier, New York, 1999), pp. 206-261, arXiv:condmat/9903101.

2 D. Jérome, in Organic Conductors: fundamentals and applications, edited by J.-P. Farges (Dekker, New York, 1994), pp. 405-494.

3 N. Dupuis, C. Bourbonnais, and J. C. Nickel, preprint cond-mat/0510544

4 V. J. Emery, R. Bruinsma, and S. Barišić, Phys. Rev. Lett. 48, 1039 (1982).

5 T. Giamarchi, Physica B230-232, 975 (1997).

6 I. E. Dzyaloshinskii and A. I. Larkin, Sov. Phys. JETP 34, 422 (1972).

7 S. Barišić and S. Brazovskii, in Recent Developments in Condensed Matter Physics, edited by J. T. Devreese (Plenum, New York, 1981), vol. 1, p. 327.

8 M. Tsuchiizu, H. Yoshioka, and Y. Suzumura, J. Phys. Soc.
Jpn. 70, 1460 (2001).

9 D. Jérome, A. Mazaud, M. Ribault, and K. Bechgaard, J. Phys. (Paris) Lett. 41, L95 (1980).

${ }^{10}$ L. Balicas, K. Behnia, W. Kang, E. Canadell, P. AubanSenzier, D. Jérome, M. Ribault, and J. M. Fabre, J. Phys. I (France) 4, 1539 (1994).

11 T. Adachi, E. Ojima, K. Kato, H. Kobayashi, T. Miyazaki, M. Tokumoto, and A. Kobayashi, J. Am. Chem. Soc. 122, 3238 (2000).

12 D. Jaccard, H. Wilhelm, D. Jérome, J. Moser, C. Carcel, and J. M. Fabre, J. Phys.: Condens. Matter 13, 89 (2001).

13 C. Bourbonnais, F. Creuzet, D. Jérome, K. Bechgaard, and A. Moradpour, J. Phys. (Paris) Lett. 45, L755 (1984).

14 P. Wzietek, F. Creuzet, C. Bourbonnais, D. Jérome, K. Bechgaard, and P. Batail, J. Phys. I (France) 3, 171 (1993).

15 V. J. Emery, in Highly Conducting One-Dimensional Solids, edited by J. T. Devreese, R. E. Evrard, and V. E. van Doren (Plenum Press, New York, 1979), p. 247. 
16 J. Sólyom, Adv. Phys. 28, 201 (1979).

17 V. J. Emery, Synthetic Metals 13, 21 (1986).

18 L. G. Caron and C. Bourbonnais, Physica 143B, 453 (1986).

19 R. Duprat and C. Bourbonnais, Eur. Phys. J. B 21, 219 (2001).

20 C. Bourbonnais and R. Duprat, J. Phys. IV France 114, 3 (2004).

21 Y. Fuseya and Y. Suzumura, J. Phys. Soc. Jpn. 74, 1264 (2005).

22 M. T. Béal-Monod, C. Bourbonnais, and V. J. Emery, Phys. Rev. B 34, 7716 (1986).

23 D. J. Scalapino, E. Loh, and J. E. Hirsch, Phys. Rev. B 34, 8190 (1986).

${ }^{24}$ C. Bourbonnais and L. G. Caron, Europhys. Lett. 5, 209 (1988).

25 H. Shimahara, J. Phys. Soc. Jpn. 58, 1735 (1989).

${ }^{26}$ H. Kino and H. Kontani, J. Phys. Soc. Jpn. 68, 1481 (1999).

27 H. Wilhelm, D. Jaccard, R. Duprat, C. Bourbonnais, D. Jérome, J. Moser, C. Carcel, and J. M. Fabre, Eur. Phys. J. B 21, 175 (2001).

28 N. Joo, P. Auban-Senzier, C. Pasquier, P. Monod, D. Jérome, and K. Bechgaard, Eur. Phys. J. B 40, 43 (2004).

29 L. P. Gorkov and D. Jérome, J. Phys. Lett. 46, L643 (1985).

${ }^{30}$ I. J. Lee, M. J. Naughton, G. M. Danner, and P. M. Chaikin, Phys. Rev. Lett. 78, 3555 (1997).

31 J. I. Oh and M. J. Naughton, Phys. Rev. Lett. 92, 67001 (2004).

${ }^{32}$ I. J. Lee, S. E. Brown, W. G. Clark, M. J. Strouse, M. J. Naughton, W. Kang, and P. M. Chaikin, Phys. Rev. Lett. 88, 17004 (2002).

33 A. G. Lebed, K. Machida, and M. Osaki, Phys. Rev. B 62, R795 (2000).

${ }^{34}$ V. Vescoli, L. Degiorgi, W. Henderson, G. Gruner, K. P. Starkey, and L. Montgomery, Science 281, 1181 (1998).

35 C. Bourbonnais and D. Jérome, Science 281, 1156 (1998).

${ }^{36}$ P. Chaikin, J. Phys. I (France) 6, 1875 (1996).

37 W. Kohn and J. M. Luttinger, Phys. Rev. Lett. 15, 524 (1965).

${ }^{38}$ K. Kuroki, R. Arita, and H. Aoki, Phys. Rev. B 63, 094509 (2001).

39 S. Onari, R. Arita, K. Kuroki, and H. Aoki, Phys. Rev. B 70, 94523 (2004).

40 Y. Tanaka and K. Kuroki, Phys. Rev. B 70, R060502 (2004).

41 L. P. Gor'kov and I. E. Dzyaloshinskii, Sov. Phys. JETP 40, 198 (1974).

42 L. Mihály and J. Sólyom, J. Low Temp. Phys. 24, 579 (1976).

43 N. Menyhárd, Sol. State Comm. 21, 495 (1977).

44 P. A. Lee, T. M. Rice, and R. A. Klemm, Phys. Rev. B 15, 2984 (1977).

${ }^{45}$ K. Šaub, S. Barišić, and J. Friedel, Phys. Lett. A 56, 302 (1976)

46 J. P. Pouget and R. Comes, in Charge Density Waves in Solids, edited by L. P. Gor'kov and G. Gruner (Elsevier Science, Amsterdam, 1989), p. 85.

47 S. Barišić and A. Bjeliš, in Theoretical Aspects of Band Structures and Electronic Properties of Pseudo-OneDimensional Solids, edited by H. Kaminura (D. Reidel, Dordrecht, 1985), p. 49.
48 J. P. Pouget and S. Ravy, J. Phys. I (France) 6, 1501 (1996).

49 J. P. Pouget and S. Ravy, Synthetic Metals 85, 1523 (1997)

50 S. Kagoshima, Y. Saso, M. Maesato, R. Kondo, and T. Hasegawa, Sol. State. Comm. 110, 479 (1999).

51 C. Bourbonnais and R. Duprat, Bulletin of the American Physical Society 49, 1:179 (2004).

52 J. C. Nickel, R. Duprat, C. Bourbonnais, and N. Dupuis, to be published in Phys. Rev. Lett. (cond-mat/0502614).

53 P. M. Grant, J. Phys. (Paris) Coll. 44, 847 (1983).

54 L. Ducasse, A. Abderraba, J. Hoarau, M. Pesquer, B. Gallois, and J. Gaultier, J. Phys. C 39, 3805 (1986).

55 C. Bourbonnais, B. Guay, and R. Wortis, in Theoretical methods for strongly correlated electrons, edited by A. M. Tremblay, D. Sénéchal, A. Ruckenstein, and C. Bourbonnais (Springer, Heidelberg, 2003), cond-mat/0204163.

56 C. Honerkamp and M. Salmhofer, Phys. Rev. B 67, 174504 (2003).

57 S. Dusuel and B. Douçot, Phys. Rev. B 67, 205111 (2003).

${ }^{58}$ C. Honerkamp, M. Salmhofer, N. Furukawa, and T. M. Rice, Phys. Rev. B 63, 35109 (2001).

59 B. Binz, D. Baeriswyl, and B. Douçot, Ann. Phys. 12, 704 (2003).

60 T. R. Morris, Int. J. Mod. Phys. 9, 2411 (1994).

61 R. Shankar, Rev. Mod. Phys. 66, 129 (1994).

62 J. C. Nickel, Ph.D. thesis, Université Paris-Sud, Orsay (2004).

63 W. H. Press, S. A. Teukolsky, W. T. Vetterling, and B. P. Flanery, Numerical Recipes in $C$ (Cambridge University Press, 1992), 2nd ed.

${ }^{64}$ L. Shampine, R. Allen, and S. Pruess, Fundamentals of Numerical Computation (John Wiley \& Sons, 1997).

65 G. Montambaux, Ph.D. thesis, Université Paris-Sud, Orsay (1985).

66 G. Montambaux, Phys. Scripta T 35, 188 (1991).

67 K. Penc and F. Mila, Phys. Rev. B 50, 11429 (1994).

${ }^{68}$ In this paper, we do not consider the case $g_{1} \simeq g_{2} \simeq$ $g_{3}$ (Hubbard model) which is characterized by a strongcoupling fixed point in the charge sector describing a 1D Mott insulator with long-range antiferromagnetic order at low temperatures due to interchain exchange interaction ${ }^{69}$.

69 C. Bourbonnais, in Les Houches, Session LVI (1991), Strongly interacting fermions and high- $T_{c}$ superconductivity, edited by B. Douçot and J. Zinn-Justin (Elsevier Science, Amsterdam, 1995), p. 307.

70 N. Dupuis and V. M. Yakovenko, Phys. Rev. B 58, 8773 (1998).

71 H. K. Ng, T. Timusk, and K. Bechgaard, Phys. Rev. B 30, 5842 (1984).

72 D. Jérome and H. Schulz, Adv. in Physics 31, 299 (1982).

73 H. Shimahara, Phys. Rev. B 61, R14936 (2000).

74 N. Cao, T. Timusk, and K. Bechgaard, J. Phys. I (France) 6, 1719 (1996)

75 D. Zanchi and A. Bjeliš, Europhys. Lett. 56, 596 (2001).

76 K. Sengupta and N. Dupuis, Phys. Rev. B 65, 035108 (2002).

77 J. C. Nickel and N. Dupuis, article in preparation.

78 E. Canadell, I.-I. Rachidi, S. Ravy, J. P. Pouget, L. Brossard, and J. P. Legros, J. Physique 50, 2967 (1989).

79 L. Brossard, M. Ribault, M. Bousseau, L. Valade, and P. Cassoux, C. R. Acad. Sc. Paris 205, 302 (1986).

${ }^{80}$ L. Brossard, M. Ribault, L. Valade, and P. Cassoux, J. 
Physique 50, 1521 (1989).

81 A. Kobayashi, H. Kim, Y. Sasaki, R. Kato, and
H. Kobayashi, Solid State Commun. 62, 57 (1987). 